\title{
CAMA
}

Centre for Applied Macroeconomic Analysis

\section{International Transmissions of Aggregate Macroeconomic Uncertainty in Small Open Economies: An Empirical Approach}

\section{CAMA Working Paper 16/2018 April 2018}

\author{
Jamie L. Cross \\ The Australian National University \\ Chenghan Hou \\ Hunan University \\ Aubrey Poon \\ University of Strathclyde \\ Centre for Applied Macroeconomic Analysis, ANU
}

\begin{abstract}
We estimate the effects of domestic and international sources of macroeconomic uncertainty in three commonly studied small open economies (SOEs): Australia, Canada and New Zealand. To this end, we propose a common stochastic volatility in mean panel VAR (CSVM-PVAR), and develop an efficient Markov chain Monte Carlo algorithm to estimate the model. Using a formal Bayesian model comparison exercise, our in-sample results suggest that foreign uncertainty spillovers shape the macroeconomic conditions in all SOEs, however domestic uncertainty shocks are important for Australia and Canada, but not New Zealand. The general mechanism is that foreign uncertainty shocks reduce real GDP and raise inflation in all SOEs, however the interest rate responses are idiosyncratic; being positive in Australia and New Zealand, and negative in Canada. Conversely, domestic uncertainty shocks tend to raise all three macroeconomic variables. Finally, in a pseudo out-of-sample forecasting exercise, the proposed model also forecasts better than traditional PVAR and CSV-PVAR benchmarks.
\end{abstract}




\section{Keywords}

Bayesian VARs, International Spillovers, State-Space Models, Stochastic Volatility in Mean, Uncertainty

\section{JEL Classification}

C11, C32, C53, E37, F69

\section{Address for correspondence:}

(E) cama.admin@anu.edu.au

ISSN 2206-0332

The Centre for Applied Macroeconomic Analysis in the Crawford School of Public Policy has been established to build strong links between professional macroeconomists. It provides a forum for quality macroeconomic research and discussion of policy issues between academia, government and the private sector.

The Crawford School of Public Policy is the Australian National University's public policy school, serving and influencing Australia, Asia and the Pacific through advanced policy research, graduate and executive education, and policy impact. 


\title{
International Transmissions of Aggregate Macroeconomic Uncertainty in Small Open Economies: An Empirical Approach ${ }^{1}$
}

\author{
Jamie L. Cross ${ }^{\mathrm{a}}$, Chenghan Hou ${ }^{\mathrm{b}, *}$, Aubrey Poon ${ }^{\mathrm{c}, \mathrm{d}}$ \\ ${ }^{a}$ Australian National University \\ ${ }^{b}$ Hunan University \\ ${ }^{c}$ University of Strathclyde \\ ${ }^{d}$ Centre for Applied Macroeconomic Analysis (CAMA)
}

\begin{abstract}
We estimate the effects of domestic and international sources of macroeconomic uncertainty in three commonly studied small open economies (SOEs): Australia, Canada and New Zealand. To this end, we propose a common stochastic volatility in mean panel VAR (CSVM-PVAR), and develop an efficient Markov chain Monte Carlo algorithm to estimate the model. Using a formal Bayesian model comparison exercise, our in-sample results suggest that foreign uncertainty spillovers shape the macroeconomic conditions in all SOEs, however domestic uncertainty shocks are important for Australia and Canada, but not New Zealand. The general mechanism is that foreign uncertainty shocks reduce real GDP and raise inflation in all SOEs, however the interest rate responses are idiosyncratic; being positive in Australia and New Zealand, and negative in Canada. Conversely, domestic uncertainty shocks tend to raise all three macroeconomic variables. Finally, in a pseudo out-of-sample forecasting exercise, the proposed model also forecasts better than traditional PVAR and CSV-PVAR benchmarks.
\end{abstract}

Keywords: Bayesian VARs, International Spillovers, State-Space Models, Stochastic Volatility in Mean, Uncertainty

JEL-Classification: C11, C32, C53, E37, F69

\footnotetext{
${ }^{1}$ We thank Professor Gary Koop, Professor Dimitris Korobilis, Professor Joshua Chan, Dr. Ben Wong and Dr. Yiqiao Sun for their comments in the development of this research.

${ }^{*}$ Corresponding author

Email addresses: j.cross@anu.edu.au (Jamie L. Cross), chenghan.hou@hotmail.com (Chenghan Hou), aubrey.poon@strath.ac.uk (Aubrey Poon)
} 


\section{Introduction}

A recent literature has demonstrated the significance of modeling macroeconomic uncertainty in the US economy (see, among others: Bloom (2009); Mumtaz and Zanetti (2013); Born and Pfeifer (2014); Fernández-Villaverde et al. (2015); Jurado et al. (2015); Baker et al. (2016); Basu and Bundick (2017); Carriero et al. (2017); Mumtaz and Theodoridis (2017a)). Since idiosyncratic shocks are the primary cause of the US business cycle, researchers tend to model the macroeconomic environment under the assumption of an autarkic state. The result is that little information is known about the effects of international uncertainty spillovers. While such information is not of first-order importance to policy makers in large economies, it is especially important for those in small open economies (SOEs); who are highly susceptible to international shocks. For instance, Justiniano and Preston (2010) suggest that around half of 2 year-ahead Canadian output growth volatility is explained by first-moment US shocks. Given the known importance of general spillovers, it is natural for policy makers to ask: what are the effects of international uncertainty spillovers in SOEs?

We address this policy-relevant question by developing a common stochastic volatility in mean (CSVM) panel VAR (PVAR). In this model, which we label CSVM-PVAR, our measures of country-specific macroeconomic uncertainty are defined as the common component in the second-moment of a particular country's macroeconomic variables. As in theoretical DSGE models, changes in the second-moment are allowed to directly impact the mean dynamics of the observable variables in our model. This enables us to empirically test the statistical significance of aggregate domestic and international sources of uncertainty in a SOE environment.

A second contribution of our paper is to develop an efficient Markov chain Monte Carlo (MCMC) sampler for estimating this new model. In traditional multivariate stochastic volatility (SV) models, such as the idiosyncratic SV model in Del Negro and Primiceri (2015), or the common SV (CSV) model in Carriero et al. (2016), the (log-)volatilities are sampled with the auxiliary mixture sampler developed in Kim et al. (1998). In our specification however, the volatilities directly enter the conditional mean equation making this procedure infeasible. To overcome this computational issue, we propose an efficient MCMC sampler that builds on recent developments in band and sparse matrix algorithms - in particular it turns out that the Hessian of the conditional density of the (log-) volatilities is a band matrix.

A third contribution of our paper is that we investigate the necessity of modeling uncertainty by conducting an in-sample Bayesian model comparison exercise, through the Bayes factor. For comprehensiveness, we also conduct an out-of-sample forecasting exercise that com- 
pares our proposed model against two commonly used benchmarks: a traditional PVAR and a CSV-PVAR, and various restricted versions of our CSVM-PVAR model. To the best of our knowledge, this is the first paper to conduct such model comparison exercises in the uncertainty literature.

In our empirical analysis we investigate the effects of domestic and international sources of uncertainty shocks in three commonly studied SOEs: Australia, Canada and New Zealand. Using the US as the large economy, and data on each country's real GDP growth, inflation and bank rates, our main results can be summarized as follows: First, the in-sample model comparison exercise suggests that while foreign uncertainty spillovers shape the macroeconomic conditions in all SOEs, domestic uncertainty is important for Australia and Canada, but not in New Zealand. Second, analysis of non-linear impulse response functions indicate that foreign uncertainty shocks tend to reduce real GDP and raise inflation in all SOEs, however the interest rate responses are idiosyncratic; being positive in Australia and New Zealand, and negative in Canada. Conversely, domestic uncertainty shocks tend to raise all three macroeconomic variables. Finally, our results from the pseudo out-of-sample forecasting exercise suggest that the proposed model forecasts better than PVAR and CSV-PVAR benchmarks.

In terms of empirical methods, our model can be viewed as a multivariate extension of the autoregressive SVM model in Koopman and Hol Uspensky (2002). Alternatively, it can be viewed as an extension of the CSV-VAR in Carriero et al. (2016), to a framework in which the time-varying second moments have first-order effects (i.e. CSVM). In this manner, it is similar to the CSVM-VAR in Mumtaz and Theodoridis (2017a). While both papers utilize a CSVM framework, we highlight four key differences between them. First, from an empirical perspective, Mumtaz and Theodoridis (2017a) analyze the US economy, while we consider the transmission of uncertainty shocks from large to small open economies. This difference in research question induces a cross-country panel dimension in the data, which can be captured through the use of a Panel VAR model. It also makes the CSVM component multivariateinstead of a scalar - which creates a non-trivial estimation problem. Second, we develop an efficient MCMC algorithm to estimate this new model. Third, we investigate the statistical importance of modeling uncertainty in our sample of countries by conducting a formal Bayesian model comparison exercise. Fourth, we also consider the importance of the CSVM component in an out-of-sample forecasting exercise.

In terms of empirical application, our research extends the wide literature on international macroeconomic spillovers (Schmitt-Grohé, 1998; Canova, 2005; Canova et al., 2007; Canova and Ciccarelli, 2012; Justiniano and Preston, 2010; Guerron-Quintana, 2013; Faccini et al., 
2016), and is intimately related to the small literature on international uncertainty spillovers (Caggiano et al., 2017; Cross et al., 2017; Mumtaz and Theodoridis, 2017b). While our research complements each of these papers, there are key differences between our paper and the already existing studies. For instance, Cross et al. (2017) employ a theoretical DSGE model in which macroeconomic uncertainty shocks are assumed to be statistically relevant and are unidirectional in nature; flowing from the US to Canada but not vice versa. In contrast, our model allows us to empirically test the significance of both domestic and international uncertainty shocks, along with the hypothesis of bidirectional spillovers - which turns out to be especially important when modeling Canada and the US. Next, Caggiano et al. (2017) utilize the economic policy uncertainty (EPU) index in Baker et al. (2016), to assess the effects of policy uncertainty spillovers from the US on Canadian unemployment over the business cycle. In contrast, we explore the general impacts of macroeconomic uncertainty by adopting a CSVM approach. This deviation in methodology is important, as recent research has shown that uncertainty indexes are prone to measurement errors; which generates bias in the associated uncertainty shock (Mertens and Ravn, 2014; Carriero et al., 2015). Finally, Mumtaz and Theodoridis (2017b) use a factor model to consider the effects of global shocks in driving macroeconomic and financial conditions in 11 OECD countries. In contrast, we focus on transmissions of country-specific uncertainty spillovers.

The rest of this paper is structured as follows. In Section 2 we introduce the CSVM-PVAR model and develop the efficient posterior simulator. In Section 3 we present the main empirical results. This includes both in-sample model selection and discussion of the effects that uncertainty shocks have in each of the SOEs, as well as a pseudo out-of-sample forecasting exercise. Finally, in Section 4 we conclude our findings and discuss some future research directions.

\section{Panel VAR with Common Stochastic Volatility in Mean}

\subsection{The Model}

In this section we introduce the Panel VAR with Common Stochastic Volatility in Mean model (CSVM-PVAR). ${ }^{2}$ To set the stage, let $\mathbf{y}_{t}=\left(\mathbf{y}_{t}^{L^{\prime}}, \mathbf{y}_{t}^{S^{\prime}}\right)^{\prime}$ denote a vector of variables of interest, where the superscripts respectively denote the set of variables in the large and small economy. In our empirical study, both $\mathbf{y}_{t}^{L}$ and $\mathbf{y}_{t}^{S}$ are $n \times 1$ vectors, however the model can

\footnotetext{
${ }^{2}$ See Canova and Ciccarelli (2013) for a nice overview of Panel VAR models, and how they differ from traditional VARs and global VARs.
} 
also accommodate vectors of distinct size. The proposed model is given by:

$$
\mathbf{y}_{t}=\mathbf{c}+\sum_{i=1}^{p} \mathbf{B}_{i} \mathbf{y}_{t-i}+\mathbf{A}\left(\begin{array}{c}
e^{h_{t}^{L}} \\
e^{h_{t}^{S}}
\end{array}\right)+\boldsymbol{\epsilon}_{t}, \quad \boldsymbol{\epsilon}_{t} \sim \mathcal{N}\left(\mathbf{0}, \boldsymbol{\Sigma}_{t}\right),
$$

where $\mathcal{N}(\cdot, \cdot)$ denotes the Gaussian distribution, $\mathbf{c}$ is a $2 n \times 1$ vector, $\mathbf{B}_{i}, i=1, \ldots, p$ are conditional mean coefficients of size $2 n$, A is a $2 n \times 2$ "uncertainty impact matrix" and the time-varying covariance matrix: $\Sigma_{t}$, is specified as:

$$
\Sigma_{t}=\left(\begin{array}{cc}
e^{h_{t}^{L}} \Sigma_{L} & 0 \\
0 & e^{h_{t}^{S}} \Sigma_{S}
\end{array}\right)
$$

where both $\Sigma_{L}$ and $\boldsymbol{\Sigma}_{S}$ are full matrices of size $n$. When estimating the model it will be convenient to express equation (1) as:

$$
\mathbf{y}_{t}=\mathbf{X}_{t} \boldsymbol{\beta}+\mathbf{A}\left(\begin{array}{c}
e^{h_{t}^{L}} \\
e^{h_{t}^{S}}
\end{array}\right)+\boldsymbol{\epsilon}_{t}, \quad \boldsymbol{\epsilon}_{t} \sim \mathcal{N}\left(\mathbf{0}, \boldsymbol{\Sigma}_{t}\right)
$$

where $\boldsymbol{\beta}=\operatorname{vec}\left(\left[\mathbf{c}, \mathbf{B}_{1}, \ldots, \mathbf{B}_{p}\right]^{\prime}\right)$ and $\mathbf{X}_{t}=\mathbf{I}_{2 n} \otimes\left(1, \mathbf{y}_{t-1}^{\prime}, \ldots, \mathbf{y}_{t-p}^{\prime}\right)$. The common log-volatilities are assumed to follow stationary $\mathrm{AR}(1)$ processes:

$$
\begin{array}{ll}
h_{t}^{L}=\rho_{L} h_{t-1}^{L}+\epsilon_{t}^{L}, & \epsilon_{t}^{L} \sim \mathcal{N}\left(0, \sigma_{L}^{2}\right), \\
h_{t}^{S}=\rho_{S} h_{t-1}^{S}+\epsilon_{t}^{L}, & \epsilon_{t}^{S} \sim \mathcal{N}\left(0, \sigma_{S}^{2}\right),
\end{array}
$$

where $\left|\rho_{i}\right|<1$ and $h_{1}^{i} \sim \mathcal{N}\left(0, \sigma_{i}^{2} /\left(1-\rho_{i}^{2}\right)\right)$ for $i \in\{L, S\}$.

The model defined in equation (2) - equation (4) can be used to investigate the effects of domestic and international sources of macroeconomic uncertainty shocks on both the small and large economies. We highlight that the covariance matrix: $\Sigma_{t}$, is changing over time, and this time-variation is driven by the common stochastic volatilities from both the large open economy: $e^{h_{t}^{L}}$, and the small open economy: $e^{h_{t}^{S}}$. As in theoretical DSGE models, these common volatilities also enter the conditional mean equation. In line with this literature, we use the volatilities as a measure of uncertainty, and refer to unanticipated changes in these volatilities as uncertainty shocks. Finally, we also highlight that the covariance matrix is block diagonal. While this assumption may be viewed as strong, in our empirical study we show that this 
parsimonious specification leads to enhanced in- and out-of-sample fit, when compared to a full covariance structure.

\subsubsection{Nested Specifications}

We highlight that our CSVM-PVAR model nests both the traditional PVAR and a common stochastic volatility PVAR (CSV-PVAR) models. As such, we can easily compare the statistical relevance of both the CSVM and CSV modeling features. In addition to these specifications, we also investigate the significance of both domestic and international uncertainty shocks by imposing four economically motivated restrictions on the impact matrix A. To ease exposition, recall that:

$$
\mathbf{A}=\left(\begin{array}{ll}
\mathbf{a}_{11} & \mathbf{a}_{12} \\
\mathbf{a}_{21} & \mathbf{a}_{22}
\end{array}\right)
$$

We consider the following four restrictions: 1) $\left.\mathbf{a}_{12}=\mathbf{0} ; 2\right) \mathbf{a}_{21}=\mathbf{0}$; 3) $\mathbf{a}_{12}=\mathbf{a}_{21}=\mathbf{0}$; 4) $\mathbf{a}_{12}=\mathbf{a}_{22}=\mathbf{0}$. Set in this manner, restriction 1 hypothesizes that uncertainty spillovers in the SOE do not transmit to the large economy; restriction 2 that uncertainty spillovers in the large economy do not transmit to the small economy; restriction 3 that uncertainty is purely idiosyncratic (i.e. no spillovers); restriction 4 that the only source of global uncertainty is from the large economy. We also highlight that the CSV-PVAR model can be viewed as a restricted version of the CSVM-PVAR with $\mathbf{A}=\mathbf{0}$.

\subsubsection{Full Covariance Structure}

To investigate the plausibility of our block diagonal covariance matrix structure, we also consider a common stochastic volatility model with full covariance structure (CSVM-PVAR-F). The measurement equation for this model is given by:

$$
\mathbf{y}_{t}=\mathbf{X}_{t} \boldsymbol{\beta}+\mathbf{A}\left(\begin{array}{c}
e^{h_{t}^{L}} \\
e^{h_{t}^{S}}
\end{array}\right)+\mathbf{B}_{0}^{-1} \boldsymbol{\epsilon}_{t}, \quad \boldsymbol{\epsilon}_{t} \sim \mathcal{N}\left(\mathbf{0}, \boldsymbol{\Sigma}_{t}\right)
$$

where $\mathbf{X}_{t}$ and $\boldsymbol{\beta}$ are defined as in equation (3), and $\mathbf{B}_{0}$ is a lower triangular matrix with ones on the main diagonal. For identification purposes, the covariance matrix is given by:

$$
\boldsymbol{\Sigma}_{t}=\left(\begin{array}{cc}
e^{h_{t}^{L}} \boldsymbol{\Omega}_{L} & \mathbf{0} \\
\mathbf{0} & e^{h_{t}^{S}} \boldsymbol{\Omega}_{S}
\end{array}\right)
$$


where $\boldsymbol{\Omega}_{L}=\operatorname{diag}\left(\sigma_{1 L}^{2}, \ldots, \sigma_{n L}^{2}\right), \boldsymbol{\Omega}_{S}=\operatorname{diag}\left(\sigma_{(n+1) S}^{2}, \ldots, \sigma_{2 n S}^{2}\right)$ and the log-volatilities follow the same processes as in equation (3) and equation (4). It is worth noting that the CSVM-PVAR considered in the previous section is a parsimonious version of the CSVM-PVAR-F model. To see this, the block diagonal covariance matrix in equation (2) can be decomposed as:

$$
\boldsymbol{\Sigma}_{t}=\left(\begin{array}{cc}
\mathbf{B}_{0,11}^{-1} & \mathbf{0} \\
\mathbf{0} & \mathbf{B}_{0,22}^{-1}
\end{array}\right)\left(\begin{array}{cc}
e^{h_{t}^{L}} \boldsymbol{\Omega}_{L} & \mathbf{0} \\
\mathbf{0} & e^{h_{t}^{S}} \boldsymbol{\Omega}_{S}
\end{array}\right)\left(\begin{array}{cc}
\mathbf{B}_{0,11}^{-1} & \mathbf{0} \\
\mathbf{0} & \mathbf{B}_{0,22}^{-1}
\end{array}\right)^{\prime}
$$

where both $\mathbf{B}_{0,11}$ and $\mathbf{B}_{0,22}$ are lower triangular matrices with ones on the main diagonal. Hence the CSVM-PVAR can be seen as a restricted version of the CSVM-PVAR-F with $\mathbf{B}_{0}$ to be the block diagonal lower triangular matrix with ones on its diagonal.

\subsubsection{Prior Specifications}

To complete the model specification, we assume independent prior distributions for each of the model parameters. We assume a Gaussian prior for the VAR coefficients and the uncertainty impact matrix: $\boldsymbol{\beta} \sim \mathcal{N}\left(\boldsymbol{\beta}_{0}, \mathbf{V}_{\beta}\right)$ and $\mathbf{a}=\operatorname{vec}(\mathbf{A})^{\prime} \sim \mathcal{N}\left(\mathbf{a}_{0}, \mathbf{V}_{a}\right)$. Moreover, the countryspecific covariance matrices for the CSVM-PVAR model are assumed to follow inverse-Wishart distributions:

$$
\Sigma_{L} \sim \mathcal{I} \mathcal{W}\left(\Phi_{L}, \delta_{L}\right), \quad \Sigma_{S} \sim \mathcal{I} \mathcal{W}\left(\Phi_{S}, \delta_{S}\right)
$$

while the $\mathrm{AR}(1)$ coefficients and variances in the state equations follow truncated normal and inverse-Gamma distributions:

$$
\begin{array}{ll}
\rho_{L} \sim \mathcal{N}\left(\rho_{L_{0}}, V_{\rho_{L}}\right) 1\left(\left|\rho_{L}\right|<1\right), & \sigma_{L}^{2} \sim \mathcal{I} \mathcal{G}\left(\nu_{L}, \gamma_{L}\right), \\
\rho_{S} \sim \mathcal{N}\left(\rho_{S_{0}}, V_{\rho_{S}}\right) 1\left(\left|\rho_{S}\right|<1\right), & \sigma_{S}^{2} \sim \mathcal{I} \mathcal{G}\left(\nu_{S}, \gamma_{S}\right)
\end{array}
$$

The $1(Q)$ is the indicator function which equals to one if statement $Q$ is true and zero otherwise. The prior distributions for $\rho_{S}$ and $\rho_{L}$ are restricted to be in the range $(-1,1)$ which implies that the $\mathrm{AR}(1)$ processes for the log-volatilities are stationary. For the CSVM-PVAR-F, let $\mathbf{b}_{0}$ be the vector stacking the parameters in each row of $\mathbf{B}_{0}$, and we assume $\mathbf{b}_{0} \sim \mathcal{N}\left(\mathbf{b}_{00}, \mathbf{V}_{b}\right)$. The diagonal elements of the $\boldsymbol{\Omega}_{L}$ and $\boldsymbol{\Omega}_{S}$ are independently distributed as

$$
\begin{aligned}
\sigma_{i L}^{2} & \sim \mathcal{I} \mathcal{G}\left(\eta_{i L}, \omega_{i L}\right), \quad i=1, \ldots, n, \\
\sigma_{j S}^{2} & \sim \mathcal{I} \mathcal{G}\left(\eta_{j S}, \omega_{i S}\right), \quad j=n+1, \ldots, 2 n
\end{aligned}
$$


We summarize all 8 models specifications in Table 1 , and defer precise details of the model comparison exercise to Section 4.1.1.

Table 1: A list of models.

\begin{tabular}{ll}
\hline \hline PVAR & constant PVAR \\
CSV-PVAR & with common SV \\
CSVM-PVAR-F & with full covariance structure \\
CSVM-PVAR & with common SVM \\
CSVM-PVAR-R1 & with common SVM with $\mathbf{a}_{12}=\mathbf{0}$ \\
CSVM-PVAR-R2 & with common SVM with $\mathbf{a}_{21}=\mathbf{0}$ \\
CSVM-PVAR-R3 & with common SVM with $\mathbf{a}_{12}=\mathbf{a}_{21}=\mathbf{0}$ \\
CSVM-PVAR-R4 & with common SVM with $\mathbf{a}_{12}=\mathbf{a}_{22}=\mathbf{0}$ \\
\hline \hline
\end{tabular}

\subsection{Bayesian Estimation}

In this section we introduce an efficient Metropolis-within-Gibbs, Markov chain Monte Carlo (MCMC) algorithm for simulating posterior draws from the CSVM-PVAR model defined in equation (2) - equation (4). We note that only minor modifications are required for the estimation of the remaining other models.

For notational convenience, let $\mathbf{y}=\left(\mathbf{y}_{1}, \ldots, \mathbf{y}_{T}\right)^{\prime}, \mathbf{h}^{L}=\left(h_{1}^{L}, \ldots, h_{T}^{L}\right)^{\prime}$ and $\mathbf{h}^{S}=\left(h_{1}^{S}, \ldots, h_{T}^{S}\right)^{\prime}$. Posterior draws can be obtained by sequentially sampling from:

1. $p\left(\mathbf{h}^{L} \mid \mathbf{h}^{S}, \mathbf{A}, \boldsymbol{\beta}, \boldsymbol{\Sigma}_{L}, \boldsymbol{\Sigma}_{S}, \rho_{L}, \rho_{S}, \sigma_{L}^{2}, \sigma_{S}^{2}, \mathbf{y}\right)=p\left(\mathbf{h}^{L} \mid \mathbf{h}^{S}, \mathbf{A}, \boldsymbol{\beta}, \boldsymbol{\Sigma}_{L}, \boldsymbol{\Sigma}_{S}, \rho_{L}, \sigma_{L}^{2}, \mathbf{y}\right)$

2. $p\left(\mathbf{h}^{S} \mid \mathbf{h}^{L}, \mathbf{A}, \boldsymbol{\beta}, \boldsymbol{\Sigma}_{L}, \boldsymbol{\Sigma}_{S}, \rho_{L}, \rho_{S}, \sigma_{L}^{2}, \sigma_{S}^{2}, \mathbf{y}\right)=p\left(\mathbf{h}^{S} \mid \mathbf{h}^{L}, \mathbf{A}, \boldsymbol{\beta}, \boldsymbol{\Sigma}_{L}, \boldsymbol{\Sigma}_{S}, \rho_{S}, \sigma_{S}^{2}, \mathbf{y}\right)$;

3. $p\left(\mathbf{A}, \boldsymbol{\beta} \mid \mathbf{h}^{L}, \mathbf{h}^{S}, \boldsymbol{\Sigma}_{L}, \boldsymbol{\Sigma}_{S}, \rho_{L}, \rho_{S}, \sigma_{L}^{2}, \sigma_{S}^{2}, \mathbf{y}\right)=p\left(\mathbf{A}, \boldsymbol{\beta} \mid \mathbf{h}^{L}, \mathbf{h}^{S}, \boldsymbol{\Sigma}_{L}, \boldsymbol{\Sigma}_{S}, \mathbf{y}\right)$;

4. $p\left(\boldsymbol{\Sigma}_{L} \mid \mathbf{h}^{L}, \mathbf{h}^{S}, \mathbf{A}, \boldsymbol{\beta}, \rho_{L}, \rho_{S}, \sigma_{L}^{2}, \sigma_{S}^{2}, \mathbf{y}\right)=p\left(\boldsymbol{\Sigma}_{L} \mid \mathbf{h}^{L}, \mathbf{A}, \boldsymbol{\beta}, \mathbf{y}\right)$;

5. $p\left(\sigma_{L}^{2} \mid \mathbf{h}^{L}, \mathbf{h}^{S}, \boldsymbol{\Sigma}_{L}, \boldsymbol{\Sigma}_{S}, \mathbf{A}, \boldsymbol{\beta}, \rho_{L}, \rho_{S}, \mathbf{y}\right)=p\left(\sigma_{L}^{2} \mid \mathbf{h}^{L}, \rho_{L}\right)$;

6. $p\left(\rho_{L} \mid \mathbf{h}^{L}, \mathbf{h}^{S}, \boldsymbol{\Sigma}_{L}, \boldsymbol{\Sigma}_{S}, \mathbf{A}, \boldsymbol{\beta}, \sigma_{L}^{2}, \sigma_{S}^{2}, \mathbf{y}\right)=p\left(\rho_{L} \mid \mathbf{h}^{L}, \sigma_{L}^{2}\right)$;

7. $p\left(\boldsymbol{\Sigma}_{S} \mid \mathbf{h}^{L}, \mathbf{h}^{S}, \mathbf{A}, \boldsymbol{\beta}, \rho_{L}, \rho_{S}, \sigma_{L}^{2}, \sigma_{S}^{2}, \mathbf{y}\right)=p\left(\boldsymbol{\Sigma}_{S} \mid \mathbf{h}^{S}, \mathbf{A}, \boldsymbol{\beta}, \mathbf{y}\right)$;

8. $p\left(\sigma_{S}^{2} \mid \mathbf{h}^{L}, \mathbf{h}^{S}, \boldsymbol{\Sigma}_{L}, \boldsymbol{\Sigma}_{S}, \mathbf{A}, \boldsymbol{\beta}, \rho_{L}, \rho_{S}, \mathbf{y}\right)=p\left(\sigma_{S}^{2} \mid \mathbf{h}^{S}, \rho_{S}\right)$;

9. $p\left(\rho_{S} \mid \mathbf{h}^{L}, \mathbf{h}^{S}, \boldsymbol{\Sigma}_{L}, \boldsymbol{\Sigma}_{S}, \mathbf{A}, \boldsymbol{\beta}, \sigma_{L}^{2}, \sigma_{S}^{2}, \mathbf{y}\right)=p\left(\rho_{S} \mid \mathbf{h}^{S}, \sigma_{S}^{2}\right)$.

The main difficulty arises in sampling from the non-standard conditional distributions of the log-volatilities in Step 1 and Step 2. Since the common stochastic volatilities: $e^{h_{t}^{S}}$ and $e^{h_{t}^{S}}$, appear in both the conditional mean and the conditional variance of the model, the efficient auxiliary mixture sampler of Kim et al. (1998) cannot be applied. In recent studies examining the impact of uncertainty on the US economy, Mumtaz and Theodoridis (2017a) sample such states using the single-move Metropolis-Hasting algorithm developed in Jacquier et al. (2002), while Carriero et al. (2017) propose a particle Gibbs sampler based on the auxiliary particle 
filter of Pitt and Shephard (1999). In this paper, we develop an efficient single-block sampler for estimating our new model. More precisely, it turns out that the Hessian of the log-conditional densities of the log-volatilities in Step 1 and Step 2 of the MCMC procedure are a band matrices. Our proposed approach therefore builds upon recent advances in band and sparse matrix algorithms (Rue et al., 2009; Chan and Jeliazkov, 2009; McCausland et al., 2011), which have been shown to perform efficiently in the estimation of state space models (Chan and Grant, 2016; McCausland, 2012; Chan, 2017). We now discuss how to sample from Steps 1-9 of the MCMC procedure.

To sample from the conditional distribution in Step 1, let:

$$
\widetilde{\mathbf{y}}_{t}=\mathbf{y}_{t}-\sum_{i=1}^{p} \mathbf{B}_{i} \mathbf{y}_{t-i}-e^{h_{t}^{S}}\left(\begin{array}{l}
\mathbf{a}_{12} \\
\mathbf{a}_{22}
\end{array}\right)
$$

where $a_{12}$ and $a_{22}$ are $n \times 1$ vectors from the uncertainty impact matrix A. Substituting equation (2) into equation (7) gives:

$$
\widetilde{\mathbf{y}}_{t}=e^{h_{t}^{L}}\left(\begin{array}{l}
\mathbf{a}_{11} \\
\mathbf{a}_{21}
\end{array}\right)+\boldsymbol{\epsilon}_{t}
$$

Thus, by a change of variable, it follows that:

$$
\begin{aligned}
p\left(\mathbf{h}^{L} \mid \mathbf{h}^{S}, \mathbf{A}_{0}, \boldsymbol{\beta}, \boldsymbol{\Sigma}_{L}, \boldsymbol{\Sigma}_{S}, \rho_{L}, \sigma_{L}^{2}, \mathbf{y}\right) & \propto p\left(\mathbf{y} \mid \mathbf{h}^{L}, \mathbf{h}^{S}, \mathbf{A}_{0}, \boldsymbol{\beta}, \boldsymbol{\Sigma}_{L}, \boldsymbol{\Sigma}_{S}\right) p\left(\mathbf{h}^{L} \mid \rho_{L}, \sigma_{L}^{2}\right) \\
& \propto p\left(\widetilde{\mathbf{y}} \mid \mathbf{h}^{L}, \mathbf{h}^{S}, \mathbf{A}_{0}, \boldsymbol{\beta}, \boldsymbol{\Sigma}_{L}, \boldsymbol{\Sigma}_{S}\right) p\left(\mathbf{h}^{L} \mid \rho_{L}, \sigma_{L}^{2}\right)
\end{aligned}
$$

The resulting $\log$-likelihood can then be written as $\log p\left(\widetilde{\mathbf{y}} \mid \mathbf{h}^{L}\right)=\sum_{t=1}^{T} \log p\left(\widetilde{\mathbf{y}}_{t} \mid h_{t}^{L}\right)$; where we suppress the conditional parameters except $\mathbf{h}^{L}$ for notational convenience. Taking a secondorder Taylor expansion around $\widetilde{\mathbf{h}}^{L}$ yields the approximation:

$$
\begin{aligned}
\log p\left(\widetilde{\mathbf{y}} \mid \mathbf{h}^{L}\right) & \approx \log p\left(\mathbf{y} \mid \widetilde{\mathbf{h}}^{L}\right)+\left(\mathbf{h}^{L}-\widetilde{\mathbf{h}}^{L}\right)^{\prime} \mathbf{f}-\frac{1}{2}\left(\mathbf{h}^{L}-\widetilde{\mathbf{h}}^{L}\right)^{\prime} \mathbf{G}\left(\mathbf{h}^{L}-\widetilde{\mathbf{h}}^{L}\right), \\
& =-\frac{1}{2}\left(\mathbf{h}^{\mathbf{L}^{\prime}} \mathbf{G} \mathbf{h}^{\mathbf{L}}-2 \mathbf{h}^{L}\left(\mathbf{f}+\mathbf{G} \widetilde{\mathbf{h}}^{L}\right)\right)+c_{1}
\end{aligned}
$$

where $c_{1}$ is a constant independent of $\mathbf{h}^{L}, \mathbf{f}=\left(f_{1}, \ldots, f_{T}\right)^{\prime}$ and $\mathbf{G}=\operatorname{diag}\left(G_{1}, \ldots, G_{T}\right)$, with

$$
f_{t}=\left.\frac{\partial}{\partial h_{t}^{L}} \log p\left(\widetilde{\mathbf{y}}_{t} \mid h_{t}^{L}\right)\right|_{h_{t}^{L}=\widetilde{h}_{t}^{L}}, \quad G_{t}=-\left.\frac{\partial^{2}}{\partial h_{t}^{L^{2}}} \log p\left(\widetilde{\mathbf{y}}_{t} \mid h_{t}^{L}\right)\right|_{h_{t}^{L}=\widetilde{h}_{t}^{L}}
$$


Thus, the log-conditional density of $\widetilde{\mathbf{y}}_{t}$ is given by:

$$
\begin{aligned}
\log p\left(\widetilde{\mathbf{y}}_{t} \mid \mathbf{h}_{t}^{L}\right) & =-\frac{n h_{t}^{L}}{2}-\frac{1}{2} e^{-h_{t}^{L}}\left(\widetilde{\mathbf{y}}_{1, t}-e^{h_{t}^{L}} \mathbf{a}_{11}\right)^{\prime} \boldsymbol{\Sigma}_{L}^{-1}\left(\widetilde{\mathbf{y}}_{1, t}-e^{h_{t}^{L}} \mathbf{a}_{11}\right) \\
& -\frac{1}{2} e^{-h_{t}^{S}}\left(\widetilde{\mathbf{y}}_{2, t}-e^{h_{t}^{L}} \mathbf{a}_{21}\right)^{\prime} \boldsymbol{\Sigma}_{S}^{-1}\left(\widetilde{\mathbf{y}}_{2, t}-e^{h_{t}^{L}} \mathbf{a}_{21}\right)
\end{aligned}
$$

It is easy to check that:

$$
\begin{aligned}
\frac{\partial}{\partial h_{t}^{L}} \log p\left(\widetilde{\mathbf{y}}_{t} \mid h_{t}^{L}\right) & =-\frac{1}{2}\left(n-e^{-h_{t}^{L}} \widetilde{\mathbf{y}}_{1, t}^{\prime} \boldsymbol{\Sigma}_{L}^{-1} \widetilde{\mathbf{y}}_{1, t}+e^{h_{t}^{L}} \mathbf{a}_{11}^{\prime} \boldsymbol{\Sigma}_{L}^{-1} \mathbf{a}_{11}\right) \\
& +e^{h_{t}^{L}-h_{t}^{S}} \widetilde{\mathbf{y}}_{2, t}^{\prime} \boldsymbol{\Sigma}_{S}^{-1} \mathbf{a}_{21}-e^{2 h_{t}^{L}-h_{t}^{S}} \mathbf{a}_{21}^{\prime} \boldsymbol{\Sigma}_{S}^{-1} \mathbf{a}_{21} \\
\frac{\partial^{2}}{\partial h_{t}^{L}} \log p\left(\widetilde{\mathbf{y}}_{t} \mid h_{t}^{L}\right) & =-\frac{1}{2}\left(e^{-h_{t}^{L}} \widetilde{\mathbf{y}}_{1, t}^{\prime} \boldsymbol{\Sigma}_{L}^{-1} \widetilde{\mathbf{y}}_{1, t}+e^{h_{t}^{L}} \mathbf{a}_{11}^{\prime} \boldsymbol{\Sigma}_{L}^{-1} \mathbf{a}_{11}\right) \\
& +e^{h_{t}^{L}-h_{t}^{S}} \widetilde{\mathbf{y}}_{2, t}^{\prime} \boldsymbol{\Sigma}_{S}^{-1} \mathbf{a}_{21}-2 e^{2 h_{t}^{L}-h_{t}^{S}} \mathbf{a}_{21}^{\prime} \boldsymbol{\Sigma}_{S}^{-1} \mathbf{a}_{21}
\end{aligned}
$$

Next, the prior density for $\mathbf{h}^{L}$ in equation (3), can be stacked over all dates $t=1, \ldots, T$ to give:

$$
\mathbf{H}_{\rho_{L}} \mathbf{h}^{L}=\boldsymbol{\epsilon}^{L}
$$

where $\boldsymbol{\epsilon}^{L}=\left(\epsilon_{1}^{L}, \ldots, \epsilon_{T}^{L}\right)$ and $\mathbf{H}_{\rho_{L}}$ is defined as follows:

$$
\mathbf{H}_{\rho_{L}}=\left(\begin{array}{ccccc}
1 & 0 & 0 & \cdots & 0 \\
-\rho_{L} & 1 & 0 & \cdots & 0 \\
0 & -\rho_{L} & 1 & \cdots & 0 \\
\vdots & \vdots & \ddots & \ddots & 0 \\
0 & 0 & \cdots & -\rho_{L} & 1
\end{array}\right)
$$

Since $\mathbf{H}_{\rho_{L}}$ is a lower triangular matrix with ones along the main diagonal it follows that $\operatorname{det}\left(\mathbf{H}_{\rho_{L}}\right)=1$, implying that $\mathbf{H}_{\rho_{L}}$ is invertible. Thus, we can write equation (12) as:

$$
\mathbf{h}^{L}=\mathbf{H}_{\rho_{L}}^{-1} \boldsymbol{\epsilon}^{L} .
$$

By a change of variable, it follows that $\mathbf{h}^{L} \sim \mathcal{N}\left(\mathbf{0},\left(\mathbf{H}_{\rho_{L}}{ }^{\prime} \mathbf{S}_{h^{L}}^{-1} \mathbf{H}_{\rho_{L}}\right)^{-1}\right)$ where $\mathbf{S}_{h^{L}}=\operatorname{diag}\left(\sigma_{L}^{2} /(1-\right.$ $\left.\left.\rho_{L}^{2}\right), \sigma_{L}^{2}, \ldots, \sigma_{L}^{2}\right)$. Hence, the log-prior density is given by:

$$
\log p\left(\mathbf{h}^{L} \mid \rho_{L}, \sigma_{L}^{2}\right)=-\frac{1}{2} \mathbf{h}^{L^{\prime}} \mathbf{H}_{\rho_{L}}^{\prime} \mathbf{S}_{h^{L}}^{-1} \mathbf{H}_{\rho_{L}} \mathbf{h}^{L}+c_{2}
$$


where $c_{2}$ is a normalization constant that is independent of $\mathbf{h}^{L}$.

Combining the log-likelihood in equation (9) with the log-prior density in equation (14) gives the log-posterior distribution:

$$
\begin{aligned}
& \log p\left(\mathbf{h}^{L} \mid \mathbf{h}^{S}, \mathbf{A}, \boldsymbol{\beta}, \boldsymbol{\Sigma}_{L}, \boldsymbol{\Sigma}_{S}, \rho_{L}, \sigma_{L}^{2}, \mathbf{y}\right) \\
= & \log p\left(\widetilde{\mathbf{y}} \mid \mathbf{h}^{L}, \mathbf{h}^{S}, \mathbf{A}, \boldsymbol{\beta}, \boldsymbol{\Sigma}_{L}, \boldsymbol{\Sigma}_{S}\right)+\log p\left(\mathbf{h}^{L} \mid \rho_{L}, \sigma_{L}^{2}\right) \\
\approx & -\frac{1}{2}\left(\mathbf{h}^{\mathbf{L}^{\prime}} \mathbf{G}^{\mathbf{L}}-2 \mathbf{h}^{L}\left(\mathbf{f}+\mathbf{G} \widetilde{\mathbf{h}}^{L}\right)\right)-\frac{1}{2} \mathbf{h}^{L^{\prime}} \mathbf{H}_{\rho_{L}}^{\prime} \mathbf{S}_{h^{L}}^{-1} \mathbf{H}_{\rho_{L}} \mathbf{h}^{L}+c_{3} \\
= & -\frac{1}{2}\left(\mathbf{h}^{\mathbf{L}^{\prime}} \mathbf{K}_{\mathbf{h}} \mathbf{h}^{\mathbf{L}}-\mathbf{h}^{\mathbf{L}^{\prime}} \mathbf{k}_{\mathbf{h}}\right)+c_{4},
\end{aligned}
$$

where $c_{3}$ and $c_{4}$ are all constant independent of $\mathbf{h}^{L}, \mathbf{K}_{\mathbf{h}}=\mathbf{G}+\mathbf{H}_{\rho_{L}}^{\prime} \mathbf{S}_{h^{L}}^{-1} \mathbf{H}_{\rho_{L}}$ and $\mathbf{k}_{\mathbf{h}}=\mathbf{f}+\mathbf{G} \widetilde{\mathbf{h}}$.

It can be seen that the above equation is the log-kernel of the Gaussian distribution. To implement the Step 1, we first set $\widetilde{\mathbf{h}}^{L}$ to be the mode of the distribution $p\left(\mathbf{h}^{L} \mid \mathbf{h}^{S}, \mathbf{A}, \boldsymbol{\beta}, \boldsymbol{\Sigma}_{L}, \boldsymbol{\Sigma}_{S}, \rho_{L}, \sigma_{L}^{2}, \mathbf{y}\right)$, which can be obtained by applying the Newton-Raphson method. The resulting Gaussian distribution $\mathcal{N}\left(\widetilde{\mathbf{h}}^{L}, \widetilde{\mathbf{K}}_{\mathbf{h}}^{-1}\right)$ is then used as our proposal in the acceptance-rejection Metropolis-Hastings step, where $\widetilde{\mathbf{K}}_{\mathbf{h}}$ is the $\mathbf{K}_{\mathbf{h}}$ evaluated at $\widetilde{\mathbf{h}}^{L}{ }^{3}$. The acceptance rates of the sampler for various common stochastic volatility in mean models listed in Table 1 are all above 85\%, which indicates that the proposed approach performs well.

Since the problem of sampling in Step 2 is symmetric to that of Step 1, it can be accomplished through a similar sampling procedure. Moreover, since Steps 3-9 of the posterior sampler are standard, we defer estimation details to Appendix Appendix A.

We close by making a few remarks on the computation. First, the common stochastic volatilities are drawn as a single block which is more efficient than a single-move sampler ${ }^{4}$. Second, due to the availability of the first and second order derivatives of the log-conditional density, the Newton-Raphson method can be used to efficiently obtain the mode of the logdensity. Lastly, since the precision matrix: $\widetilde{\mathbf{K}}_{\mathbf{h}}$, is a band matrix, we can efficiently obtain draws from the proposal distribution by applying the precision sampler in Chan and Jeliazkov (2009).

\footnotetext{
${ }^{3}$ As seen in equation (11), there is no guarantee that $\widetilde{\mathbf{K}}_{\mathbf{h}}$ is a positive definite matrix. To overcome this problem we adopt the following strategy: First, we initialize $\widetilde{\mathbf{K}}_{\mathbf{h}}$ as an identity matrix. Next, in each MCMC iteration, we check whether the proposed Hessian $\widetilde{\mathbf{K}}_{\mathbf{h}}$ is positive definite. If it is, then we use it in the proposal distribution, otherwise we use the $\widetilde{\mathbf{K}}_{\mathbf{h}}$ from the previous MCMC iteration.

${ }^{4}$ In general, single-block sampler is shown to be more numerically efficient than single-move sampler when the posterior samples are highly correlated.
} 


\section{Data and Priors}

The data for each country consists of quarterly data on real GDP, CPI inflation and a short-term interest rate - taken to be the country's bank-rate - from 1978Q3-2016Q4. All series were sourced from the International Monetary Fund's (IMF's) International Financial Statistics (IFS) database. Before entering the model, both real GDP and CPI indexes were converted to annualized growth measures.

To conduct the analysis, we set a Minnesota type of prior for the VAR coefficients. In particular, the prior mean is set to a zero vector, $\boldsymbol{\beta}_{0}=\mathbf{0}$, and the prior covariance matrix is diagonal with its corresponding elements set as follows:

$$
\begin{aligned}
& \operatorname{Var}(\mathbf{c})=100 \times \mathbf{I}_{2 n}, \\
& \operatorname{Var}\left(\mathbf{B}_{l}^{i j}\right)= \begin{cases}\frac{\lambda_{1}^{2} \lambda_{2}}{l^{\lambda_{3}}} \frac{\sigma_{i}}{\sigma_{j}} & \text { for } l=1, \ldots, p \text { and } i \neq j, \\
\frac{\lambda_{1}^{2}}{l^{\lambda_{3}}} & \text { for } l=1, \ldots, p \text { and } i=j,\end{cases}
\end{aligned}
$$

where $\mathbf{B}_{l}^{i j}$ denotes the $(i, j)$ th element of the matrix $\mathbf{B}_{l}$ and $\sigma_{r}$ is set equal to the standard deviation of the residual from $\operatorname{AR}(p)$ model for the variable $r$. The hyperparameters are set to be $\lambda_{1}=0.2, \lambda_{2}=0.5, \lambda_{3}=2$. We assume a relatively non-informative prior on the uncertainty impact matrix. To be specific, we set $\mathbf{a}_{0}=\mathbf{0}$ and $\mathbf{V}_{a}=5 \times \mathbf{I}_{2 n}$. For the CSVM-PVAR model, the degree of freedom parameters of the covariance matrices are set to $\delta_{S}=n+4$ and $\delta_{L}=n+4$, and the scale matrices $\Phi_{L}=\left(\delta_{L}-n-1\right) \mathbf{I}_{n}$ and $\Phi_{S}=\left(\delta_{S}-n-1\right) \mathbf{I}_{n}$. These values imply that the expected value of $\boldsymbol{\Sigma}_{L}$ and $\boldsymbol{\Sigma}_{S}$ are equal to identity matrices of size $n$. We also set $\nu_{L}=10, \nu_{S}=10, \gamma_{L}=0.05\left(\nu_{L}-1\right)$ and $\gamma_{S}=0.05\left(\nu_{S}-1\right)$. These hyperparameters imply that the prior mean of $\sigma_{L}^{2}$ and $\sigma_{S}$ are both equal to 0.05 . For the autoregressive coefficient for the log-volatility, we set $\rho_{L_{0}}=\rho_{S_{0}}=0.9$ and $V_{\rho_{L}}=V_{\rho_{S}}=0.2^{2}$. For the CSVM-PVAR-F model, we set $\eta_{i L}=\eta_{j L}=10$ and $\omega_{i L}=\omega_{i S}=9$ for $i, j=1, \ldots, n$. This implies that $\mathbb{E}\left(\sigma_{i L}^{2}\right)=\mathbb{E}\left(\sigma_{i S}^{2}\right)=1$. Lastly, we let $\mathbf{b}_{00}=\mathbf{0}$ and $\mathbf{V}_{b}=5 \mathbf{I}_{2 n(n-1)}$. All posterior estimates in our empirical results are based on 55000 posterior draws from the MCMC method after a burnin-in-period of 5000 draws.

\section{Empirical Results}

In this section we discuss our main empirical results on the effects of domestic and international macroeconomic uncertainty spillovers between the US and three commonly studied SOEs: Australia, Canada and New Zealand. To facilitate our discussion, we split the results 
into two sub-sections: In-sample and out-of-sample analysis. To maintain consistency with the broader uncertainty literature, we select a lag length of $p=2$ for the PVAR coefficients (e.g., Caggiano et al. (2017); Carriero et al. (2017); Mumtaz and Theodoridis (2017b)).

\subsection{In-Sample Analysis}

Before examining the effects of uncertainty shocks, we first demonstrate the ability of our model to capture the dynamics of each country's data. We do so by conducting a formal Bayesian model comparison exercise, via the Bayes factor. Having selected the best model, we then discuss the qualitative properties of our proposed uncertainty measures. Finally, we address our main empirical question by investigating both the direct and indirect effects of the domestic and international uncertainty shocks in each SOE.

\subsubsection{Model Selection}

To identify the significance of modeling uncertainty in each of the SOEs, we compare insample statistics of the proposed CSVM-PVAR model against the seven alternative model specifications in Table 1. Since we employ Bayesian estimation, the natural metric for in-sample fit is the Bayes factor - a special case of the posterior odds ratio. To illustrate this procedure, let $M_{1}$ and $M_{2}$ denote two arbitrary models. The posterior odds ratio for $M_{1}$ against $M_{2}$, is defined as:

$$
P O_{1,2}=\frac{\mathcal{P}\left(M_{1} \mid \mathbf{y}^{\mathbf{o}}\right)}{\mathcal{P}\left(M_{2} \mid \mathbf{y}^{\mathbf{o}}\right)}
$$

where $\mathcal{P}\left(M_{i} \mid \mathbf{y}^{\mathbf{o}}\right)$ denotes the (conditional) probability of $M_{i}, i=1,2$, given the observed data: $\mathbf{y}^{o}=\left(\mathbf{y}_{1}^{o}, \ldots, \mathbf{y}_{T}^{o}\right)$. By the law of conditional probability, the posterior odds ratio can be written as:

$$
P O_{1,2}=\frac{p\left(\mathbf{y}^{\mathbf{o}} \mid M_{1}\right)}{p\left(\mathbf{y}^{\mathbf{o}} \mid M_{2}\right)} \times \frac{\mathcal{P}\left(M_{1}\right)}{\mathcal{P}\left(M_{2}\right)}
$$

where $p\left(\mathbf{y} \mid M_{i}\right)$ and $\mathcal{P}\left(M_{i}\right)$ respectively denote the marginal likelihood and prior model probability for $M_{i}, i=1,2$, where the marginal likelihood is defined as:

$$
p\left(\mathbf{y}^{\mathbf{o}} \mid M_{i}\right)=\int_{\boldsymbol{\Theta}_{i}} p\left(\mathbf{y}^{\mathbf{o}} \mid \boldsymbol{\theta}_{i}, M_{i}\right) p\left(\theta_{i} \mid M_{i}\right) d \boldsymbol{\theta}_{i}
$$

where $\boldsymbol{\theta}_{i}$ is a vector of the parameters in model $M_{i}$, and $\boldsymbol{\Theta}_{i}$ is the associated parameter space. To compute this integral, we use the fact that it can be represented as a product of one-stepahead predictive likelihoods evaluated at the observed data (Geweke and Amisano, 2011). That 
is:

$$
p\left(\mathbf{y}^{\mathbf{o}} \mid M_{i}\right)=p\left(\mathbf{y}_{1}^{o} \mid M_{i}\right) \prod_{t=2}^{T} p\left(\mathbf{y}_{t}^{o} \mid \mathbf{y}_{1}^{o}, \ldots, \mathbf{y}_{t-1}^{o}, M_{i}\right)
$$

The ratio of two such likelihoods is known as the Bayes factor. It can easily be seen that the posterior odds ratio reduces to the Bayes factor of $M_{1}$ against $M_{2}$, denoted $B F_{1,2}$, under the assumption of equal prior model probabilities (i.e. $\mathcal{P}\left(M_{1}\right)=\mathcal{P}\left(M_{2}\right)$ ). Since it is a special case of the posterior odds ratio, the Bayes factor takes on a probabilistic interpretation. For instance, if $B F_{1,2}=2$ then $M_{1}$ is twice as likely as $M_{2}$ given the data.

The results for each of the models in Table 1 are provided in Table B.2 in Appendix B.1. The general conclusion is that the (log-)marginal likelihoods provide overwhelming support in favor of our class of CSVM-PVAR models against the PVAR and CSV-PVAR models across all countries. For instance, in the case of Australia, the Bayes factors for the CSVM-PVARR1 against the CSV-PVAR is approximately 1 million (i.e. $\exp (-903.64+917.47)$ ). More specifically, the plain CSVM-PVAR provides the best fit for Canada, the CSVM-PVAR-R1 variant for Australia, and the CSVM-PVAR-R4 for New Zealand. These results suggest that while international uncertainty spillovers are a key feature of the macroeconomic environment in each of the SOEs, domestic uncertainty is important in both Australia and Canada, but not in New Zealand. Interestingly, selection of the CSVM-PVAR in Canada indicates that uncertainty spillovers between the US and Canada are bilateral in nature. In contrast, the selected model variants for Australia and New Zealand indicate that uncertainty spillovers are unilateralflowing from the US to the SOE but not vice versa. Finally, we note that the CSVM-PVAR model outperforms the more general CSVM-PVAR-F model, thus providing empirical support for the diagonal covariance matrices in the CSVM-PVAR model.

\subsubsection{Aggregate Uncertainty Measures}

Having identified the best models for the in-sample analysis, we now discuss the qualitative behavior of the implied measures of uncertainty. To this end, Figures B.1 and B.2 in Appendix B.2 present the posterior distributions of the macroeconomic uncertainty index for both Australia and Canada. In each figure, the blue line represents the posterior mean, and the red lines represent the $90 \%$ credible set. Since the model comparison section revealed that domestic macroeconomic uncertainty is not statistically relevant for New Zealand, no such figure is presented.

The broad differences in the two macroeconomic uncertainty measures are particularly striking. Uncertainty in Australia is relatively smooth, starts high and then declines over the sample period. In contrast, uncertainty in Canada tends to fluctuate around a constant mean. 
On an individual level, uncertainty in Australia increases following the 1979 energy crisis, before declining after the movement to a flexible exchange rate regime in 1983. The subsequent spike in the late 1980s is likely due to uncertainty surrounding the international "Black Monday" stock market crash of October 19, 1987 and subsequent recession. Interestingly, the adoption of inflation targeting in the early 1990s seems to coincide with the decline in uncertainty over the next two decades. The notable spike in the early 2000's likely relates to the "Dot-Com bubble". Finally, the relatively mild spike in 2008 corresponds to the Global Financial Crisis (GFC).

Similar to Australia, uncertainty in Canada increases following the 1979 energy crisis before returning to baseline by the mid-1980s, where it remained for the duration of the Great Moderation, ending in 2007. Two notable spikes in this period surround the recessions of 1990 and the early 2000s. In contrast to the Australian case, the spike in uncertainty during the GFC is the largest in Canada's history. This result will be emphasized in the next section, where we show that Canada and the US are highly linked, while Australia's responsiveness to US shocks declines over the sample period. Finally, the uncertainty measure spikes again around the 2015 recession.

\subsubsection{Effects of uncertainty shocks}

What effect do international macroeconomic spillovers have on a SOE? To answer this question, we first consider the estimated impact matrix of the implied best model in Section 4.1.1, and then analyze generalized impulse response functions (GIRFs). ${ }^{5}$ Knowledge of the impact matrix is useful as it highlights the sign and magnitude effects of an uncertainty shocks in the initial period. In this sense, we can measure the direct effects of both domestic and international sources of uncertainty shocks. Moreover, GIRFs are useful as they provide information about the how uncertainty shock propagate throughout the economy. In this sense, we can also measure the indirect effects of the uncertainty shocks. The various estimates of the impact matrix and GIRFs are presented in Appendix B.3. We now discuss each in turn.

The columns of Tables B.3-B.5 respectively represent the US and SOE uncertainty shock, while the rows represent the various macroeconomic variables in the US and SOEs: real GDP growth, CPI inflation and interest. To ease exposition we have divided the matrix into four quadrants. The first quadrant represents the impact of spillovers from the SOE shock on the US; the second quadrant the US shock on itself; the third quadrant spillovers from the US to the

\footnotetext{
${ }^{5}$ Since the measures of aggregate uncertainty are time varying, we follow Koop et al. (1996) and compute GIRFs. The difference between GIRFs and traditional IRFs, is that future shocks are not "zeroed-out" by assumption, but instead "integrated-out" through a Monte Carlo integration procedure, details of which are provided in Koop et al. (1996).
} 
SOE; and the fourth quadrant the SOE on itself. For instance, in the case of New Zealand (see Table B.5), the CSVM-PVAR-R4 model has no domestic uncertainty shocks, implying that the second column is all zeros. Similarly, in the case of Australia (Table B.3), the CSVM-PVAR-R1 model has no direct uncertainty spillovers from Australia to the US, implying all entries in the first quadrant are zero.

A few general observations can be made. First, international uncertainty spillovers have negative impacts on real GDP and interest, however the inflation response is positive. While this result is novel for the set of SOEs used in our study, they are broadly consistent with those in Mumtaz and Theodoridis (2015); who find that uncertainty shocks from the US negatively effect UK real GDP and interest, but positive impact inflation. Second, the impact of domestic uncertainty shocks in Australia and Canada are all positive. Third, in line with the broad literature on the US economy, we find that domestic uncertainty shocks decrease output while increasing inflation and interest rates (Born and Pfeifer, 2014; Mumtaz and Theodoridis, 2015; Carriero et al., 2017; Mumtaz and Theodoridis, 2017a).

While the impact matrix provides information about the direct transmission of uncertainty shocks, it is silent on how such shocks propagate throughout the economy. To further investigate the effects of uncertainty shocks over our sample period, we now discuss the GIRFs in Figures B.3-B.7. In each figure, the curves represent mean point estimates to a (time-varying) one-standard deviation shock of the log-uncertainty measures. Subplots in rows respectively represent the US and SOE response to a given uncertainty shock, while those in columns represent the macroeconomic variables. Finally, the x-axis represents an impulse horizon of 20 quarters (5 years), the y-axis shows the sample dates and the z-axis is percentage point changes. Since there are general differences in the transmission of US shocks throughout the SOEs, we briefly discuss each country's responses in turn.

The Australian inflation and real GDP responses to a US uncertainty shock are qualitatively similar to those in the US, however the interest rate moves in the opposite direction. Also, the magnitudes of the Australian responses are about half the size of those in the US. For instance, following a US uncertainty shock, the peak real GDP response in the US is about -2 percent, compared to -1 percent in Australia. Similarly, following an uncertainty shock from Australia, the peak inflation response in the US is about 0.4 percent, compared to 1.5 percent in Australia. In contrast, the effects of domestic uncertainty shocks are much smaller and are also decreasing over the sample period. Note that despite having zero direct impact on the US economy, uncertainty shocks from Australia indirectly affect the US through changes in the Australian macroeconomic variables. That being said, these impacts are generally small (less than 25 basis 
points).

Next, the GIRFs between Canada and the US are both qualitatively and quantitatively similar. This suggests close links between Canada and the US economies. Interestingly, while the inflation and interest rates remain positive over the entire impulse horizon, the contemporaneous impact of a US shock is negative, but quickly rebounds to be positive. This result is in line with Bloom (2009) who finds that uncertainty shocks in the US tend to generate short, sharp recessions and recoveries. In contrast, uncertainty shock from Canada tend to have a long lasting reduction in each variable. Importantly, the finding that uncertainty impacts from Canada has a large impact on the US suggests that bi-lateral uncertainty transmissions between Canada and US are important.

Finally, in the case of New Zealand, the US inflation and real GDP responses are similar to those seen in Australia and Canada. As in Australia, the New Zealand inflation and real GDP responses tend to follow those in the US, however the interest rate respnses are unique. Moreover, while the inflation responses are almost identical, the real GDP responses are approximately half the size. Since the CSVM-PVAR-R4 model restricts uncertainty shocks to the US, there is no corresponding set of domestic impulse responses for New Zealand.

\subsection{Forecasting Results}

In addition to in-sample model comparison, we also conduct an out-of-sample forecasting exercise. In this step, we compare the ability of each model in Table 1 to predict key macroeconomic variables: real GDP, inflation and the short-term interest rate, of the various SOEs in our study. We evaluate the iterated $h$-step-ahead forecast of each model with $h=1,2,4,8$, and the forecast evaluation period is from 1990Q1 - 2016Q4.

To assess the point forecast accuracy we report both the root mean squared forecast error (RMSFE) and the mean absolute forecast error (MAFE):

$$
\begin{gathered}
\mathrm{RMSFE}=\sqrt{\frac{\sum_{t=t_{0}}^{T-h}\left(\mathbf{y}_{t+h}^{o}-\mathbb{E}\left(\mathbf{y}_{t+h} \mid \mathbf{y}_{1: t}\right)\right)^{2}}{T-h-t_{0}+1}}, \\
\mathrm{MAFE}=\frac{\sum_{t=t_{0}}^{T-h}\left|\mathbf{y}_{t+h}^{o}-\hat{\mathbf{y}}_{t+h}^{M}\right|}{T-h-t_{0}+1},
\end{gathered}
$$


where $\mathbb{E}\left(\mathbf{y}_{t+h} \mid \mathbf{y}_{1: t}\right)$ is the posterior mean of the predictive density and $\hat{\mathbf{y}}_{t+h}^{M}$ is the posterior median of the predictive density.

In addition to point forecasts, we also compute density forecasts for each model. To evaluate these forecasts, we report the average log-predictive likelihoods (ALPL) and the average continuous rank probability score (ACRPS):

$$
\begin{gathered}
\mathrm{ALPL}=\frac{\sum_{t=t_{0}}^{T-k} \log p_{t+h}\left(\mathbf{y}_{t+k}=\mathbf{y}_{t+h}^{o} \mid \mathbf{y}_{1}^{o}, \ldots, \mathbf{y}_{t-1}^{o}\right)}{T-h-t_{0}+1}, \\
\mathrm{ACRPS}=\frac{1}{T-h-t_{0}+1} \sum_{t=t_{0}}^{T-h} \mathrm{CRPS}_{t}
\end{gathered}
$$

where $\operatorname{CRPS}_{t}=\int_{-\infty}^{\infty}\left(F_{t+h}(z)-1\left(\mathbf{y}_{t+h}^{o}<z\right)\right)^{2} d z=\mathbb{E}_{p_{t+h}}\left|\mathbf{y}_{t+h}-\mathbf{y}_{t+h}^{o}\right|-0.5 \mathbb{E}_{p_{t+h}}\left|\mathbf{y}_{t+h}-\mathbf{y}_{t+h}^{\prime}\right|$ and $F_{t+h}$ is the cumulative distribution of the predictive dnsity at time $t+h$ given all information up to time $t$. the A small the value of the ACRPS indicates a better forecasting performance.

The point and density forecast results for each of the models in Table 1 are reported in Appendix B.4. To facilitate comparison, we report relative scores to a PVAR benchmark. Set in this manner, a relative RMSFE, MAFE and ACRPS of less than one indicates that the given model provides a better forecast than the PVAR benchmark. Conversely, a positive value for the relative ALPL indicates a better forecasting performance than the benchmark.

While there is no strictly dominant model for any country or variable, the general trend is that models with CSVM components forecast better than the alternatives. In fact, with just one exception, the CSVM-PVAR models provide better forecasts than both the PVAR and CSV-PVAR. This exception is real GDP in New Zealand, for which the simple PVAR model tends to produce the best point forecasts, however the CSVM component is particularly useful at one-step-ahead prediction and dominates the PVAR when conducting density forecasts. Combined with the in-sample analysis, our forecasting results provide overwhelming support for our proposed CSVM-PVAR model - or one of its restrictions - against both the traditional PVAR and CSV-PVAR models. Economically, this suggests that uncertainty spillovers play a key role in shaping the macroeconomic environments in all SOEs.

\section{Concluding Remarks and Future Research}

In this paper, we estimated the effects of domestic and international sources of macroeconomic uncertainty in three commonly studied small open economies (SOEs): Australia, Canada 
and New Zealand. To this end, we proposed a common stochastic volatility in mean panel VAR (CSVM-PVAR) model. To estimate the model, we developed an efficient Markov chain Monte Carlo algorithm that built upon recent advances in band and sparse matrix algorithms. Our results showed that foreign uncertainty spillovers shape the macroeconomic conditions in all SOEs, however domestic uncertainty shocks are important for Australia and Canada, but not New Zealand. The general mechanism is that foreign uncertainty shocks reduce real GDP and raise inflation in all SOEs, however the interest rate responses are idiosyncratic; being positive in Australia and New Zealand, and negative in Canada. Conversely, domestic uncertainty shocks tend to raise all three macroeconomic variables. Finally, in a pseudo out-of-sample forecasting exercise, the proposed model also forecasts better than traditional PVAR and CSV-PVAR benchmarks.

In closing, we point towards three avenues for future research. First, it would be interesting to investigate whether the international transmission mechanism of the identified uncertainty shocks is changing over time. Second, given the recent surge of interest surrounding financial versus macroeconomic shocks, it would be useful to investigate transmissions of financial uncertainty from large to small open economies. Finally, given the empirical relevance of uncertainty spillovers from Canada to the US, it would be useful to extend the US literature by investigating the transmission mechanism in an open economy DSGE model.

\section{Appendix A. Technical Appendix}

In this Appendix we explain how to obtain draws from Steps 3-9 of the posterior sampler in Section 2.2. To this end, given $\mathbf{h}^{L}$ and $\mathbf{h}^{S}$, we first write Equation (2) as:

$$
\mathbf{y}_{t}=\mathbf{X}_{t} \boldsymbol{\beta}+\widetilde{\mathbf{Z}}_{t} \mathbf{a}+\boldsymbol{\epsilon}_{t} \quad \boldsymbol{\epsilon}_{t} \sim \mathcal{N}\left(\mathbf{0}, \boldsymbol{\Sigma}_{t}\right)
$$

where $\mathbf{Z}_{t}=\mathbf{I}_{2 n} \otimes\left(e^{h_{t}^{L}}, e^{h_{t}^{S}}\right)$ and $\mathbf{a}=\operatorname{vec}\left(\mathbf{A}^{\prime}\right)$. Stacking the above equation over $t=1, \ldots, T$, we get:

$$
\mathbf{y}=\mathrm{Z} \gamma+\boldsymbol{\epsilon}, \quad \boldsymbol{\epsilon} \sim \mathcal{N}(\mathbf{0}, \mathbf{\Sigma})
$$


where $\mathbf{y}=\left(\mathbf{y}_{1}, \ldots, \mathbf{y}_{T}\right)^{\prime}, \boldsymbol{\gamma}=\left(\boldsymbol{\beta}^{\prime}, \boldsymbol{\gamma}^{\prime}\right)^{\prime}, \boldsymbol{\Sigma}=\mathbf{I}_{2 n} \otimes \boldsymbol{\Sigma}_{t}$ and:

$$
\mathbf{Z}=\left(\begin{array}{cc}
\mathbf{X}_{1} & \widetilde{\mathbf{Z}}_{1} \\
\vdots & \vdots \\
\mathbf{X}_{T} & \widetilde{\mathbf{Z}}_{T}
\end{array}\right)
$$

For the Step 3 of the sampler, let $\mathbf{V}_{\boldsymbol{\gamma}}=\operatorname{diag}\left(\mathbf{V}_{\beta}, \mathbf{V}_{a}\right)$ and $\boldsymbol{\gamma}_{0}=\left(\boldsymbol{\beta}_{0}^{\prime}, \mathbf{a}_{0}^{\prime}\right)^{\prime}$, then using the standard results from linear regression, we have:

$$
\left(\mathbf{A}, \boldsymbol{\beta} \mid \mathbf{h}^{L}, \mathbf{h}^{S}, \boldsymbol{\Sigma}_{L}, \mathbf{\Sigma}_{S}, \mathbf{y}\right) \equiv\left(\gamma \mid \mathbf{h}^{L}, \mathbf{h}^{S}, \boldsymbol{\Sigma}_{L}, \boldsymbol{\Sigma}_{S}, \mathbf{y}\right) \sim \mathcal{N}\left(\widehat{\gamma}, \mathbf{D}_{\gamma}\right)
$$

where $\mathbf{D}_{\gamma}^{-1}=\mathbf{Z}^{\prime} \boldsymbol{\Sigma}^{-1} \mathbf{Z}+\mathbf{V}_{\gamma}^{-1}$ and $\widehat{\gamma}=\mathbf{D}_{\gamma}\left(\mathbf{Z}^{\prime} \boldsymbol{\Sigma}^{-1} \mathbf{y}+\mathbf{V}_{\gamma}^{-1} \boldsymbol{\gamma}_{0}\right)$.

To implement Step 4 - Step 6, we first collect the first $n$ equations in (A.1) to get:

$$
\mathbf{y}_{t}^{L}=\mathbf{X}_{t}^{L} \boldsymbol{\beta}+\widetilde{\mathbf{Z}}_{t}^{L} \mathbf{a}+\boldsymbol{\epsilon}_{t}^{L} \quad \boldsymbol{\epsilon}_{t}^{L} \sim \mathcal{N}\left(\mathbf{0}, e^{h_{t}^{L}} \boldsymbol{\Sigma}_{L}\right)
$$

Then, for Step 4, we have:

$$
\left(\boldsymbol{\Sigma}_{L} \mid \mathbf{h}^{L}, \mathbf{A}, \boldsymbol{\beta}, \mathbf{y}\right) \sim \mathcal{I} \mathcal{W}\left(\widehat{\Phi}_{L}, \widehat{\delta}_{L}\right)
$$

where $\widehat{\Phi}_{L}=\Phi_{L}+\sum_{t=1}^{T} e^{-h_{t}^{L}}\left(\mathbf{y}_{t}^{L}-\mathbf{X}_{t}^{L} \boldsymbol{\beta}-\widetilde{\mathbf{Z}}_{t}^{L} \mathbf{a}\right)\left(\mathbf{y}_{t}^{L}-\mathbf{X}_{t}^{L} \boldsymbol{\beta}-\widetilde{\mathbf{Z}}_{t}^{L} \mathbf{a}\right)^{\prime}$ and $\widehat{\delta}_{L}=\delta_{L}+T$.

In Step 5, the conditional conjugate prior on the variance of the log-volatilities yields an Inverse-gamma distribution:

$$
\left(\sigma_{L}^{2} \mid \mathbf{h}^{L}, \rho_{L}\right) \sim \mathcal{I} \mathcal{G}\left(\nu_{L}+\frac{T}{2}, \hat{\gamma}_{L}\right)
$$

where $\hat{\gamma}=\frac{1}{2}\left(\left(1-\rho_{L}^{2}\right) h_{1}^{L^{2}}+\sum_{t=2}^{T}\left(h_{t}^{L}-\rho_{h} h_{t-1}^{L}\right)^{2}\right)$. For Step 6, we implement an indepdencechain Metroplis-Hasting step with a truncated Gaussian proposal distribution bounded between -1 and 1 . The mean of the proposal distribution is set to be the mode of the full conditional distributin, which can be obtained using the Newton-Raphson method, and the variance of the proposal distribution is set to be the inverse of the negative Hessian evaluated at the mode. Finally, the implementation for Steps 7-9 are similar to those estimation procedures in Steps $4-6$.

Drawing the log-volatilities form the CSVM-PVAR-F is similar to those for the CSVM- 
PVAR. To see this, we first multiply $\mathbf{B}_{0}$ to both sides of the equation (5) and rewrite it as

$$
\widetilde{\widetilde{\mathbf{y}}}_{t}=\widetilde{\mathbf{X}}_{t} \boldsymbol{\beta}+\widetilde{\mathbf{A}}\left(\begin{array}{c}
e^{h_{t}^{L}} \\
e^{h_{t}^{S}}
\end{array}\right)+\boldsymbol{\epsilon}_{t}, \quad \boldsymbol{\epsilon}_{t} \sim \mathcal{N}\left(\mathbf{0}, \boldsymbol{\Sigma}_{t}\right)
$$

where $\widetilde{\widetilde{\mathbf{y}}}_{t}=\mathbf{B}_{0} \mathbf{y}_{t}, \widetilde{\mathbf{X}}_{t}=\mathbf{B}_{0} \mathbf{X}_{t}$ and $\widetilde{\mathbf{A}}=\mathbf{B}_{0} \mathbf{A}$. The covariance matrix $\boldsymbol{\Sigma}_{t}$ is defined as in the equation (6) which is a (block) diagonal matrix. It can be seen that the equation (A.3) is in the same form as the equation (2), thus the draws from the full conditional distribution of the $\log$-volatilities $\mathbf{h}^{L}$ and $\mathbf{h}^{S}$ can be obtained using the efficient approach proposed in this paper. Given the log-volatilites, the posterior draws for $(\boldsymbol{\beta}, \mathbf{A})$ can be obtained similarly as in (A.2).

For sampling $\mathbf{B}_{0}$, we can first rearrange the equation (5) as

$$
\mathbf{B}_{0} \mathbf{y}_{t}^{*}=\boldsymbol{\epsilon}_{t}, \quad \boldsymbol{\epsilon}_{t} \sim \mathcal{N}\left(\mathbf{0}, \Sigma_{t}\right),
$$

where $\mathbf{y}_{t}^{*}=\left(y_{1, t}^{*}, \ldots, y_{2 n, t}^{*}\right)^{\prime}$ and it is defined as

$$
\mathbf{y}_{t}^{*}=\mathbf{y}_{t}-\mathbf{X}_{t} \boldsymbol{\beta}+\mathbf{A}\left(\begin{array}{c}
e^{h_{t}^{L}} \\
e^{h_{t}^{S}}
\end{array}\right)
$$

Since the $\mathbf{B}_{0}$ is a lower triangular matrix with ones in its diagonal, it can be seen that the equation (A.4) can be rewritten into a form of standard linear regression model:

$$
\mathbf{y}_{t}^{*}=\mathbf{W}_{t} \mathbf{b}_{0}+\boldsymbol{\epsilon}_{t}, \quad \boldsymbol{\epsilon}_{t} \sim \mathcal{N}\left(\mathbf{0}, \boldsymbol{\Sigma}_{t}\right),
$$

where $\mathbf{b}_{0}$ is a column vector stacking the parameters in each row of $\mathbf{B}_{0}$ and

$$
\mathbf{W}_{t}=\left(\begin{array}{ccccccc}
0 & 0 & 0 & \cdots & \cdots & \cdots & 0 \\
-y_{1, t}^{*} & 0 & 0 & \cdots & \cdots & \cdots & 0 \\
0 & -y_{1, t} & -y_{2, t}^{*} & \cdots & \cdots & \cdots & 0 \\
\vdots & \vdots & \ddots & \vdots & \cdots & \cdots & 0 \\
0 & \cdots & \cdots & -y_{1, t}^{*} & -y_{2, t}^{*} & \cdots & -y_{2 n-1, t}^{*}
\end{array}\right) .
$$

Thus, standard results for the linear regression model can be applied to obtain draws of $\mathbf{b}_{0}$. The full conditional distributions for the variance of the shock is standard. In particular, it 
follows

$$
\begin{aligned}
& \sigma_{i L}^{2} \sim \mathcal{I} \mathcal{G}\left(\frac{T}{2}+\eta_{i L}, \omega_{i L}+\sum_{t=1}^{T} d y_{i, t}^{2} e^{-h_{t}^{L}}\right), \quad i=1, \ldots, n \\
& \sigma_{j S}^{2} \sim \mathcal{I} \mathcal{G}\left(\frac{T}{2}+\eta_{j S}, \omega_{j S}+\sum_{t=1}^{T} d y_{j, t}^{2} e^{-h_{t}^{S}}\right), \quad j=n+1, \ldots, 2 n,
\end{aligned}
$$

where $d y_{i, t}$ is the $j$ th element of the vector $\mathbf{y}_{t}^{*}-\mathbf{W}_{t} \mathbf{b}_{0}$.

\section{Appendix B. Tables and Figures}

Appendix B.1. Marginal Likelihood Results

Table B.2: Estimated log marginal likelihoods for various models in Table 1.

\begin{tabular}{lccc}
\hline \hline & Australia & Canada & New Zealand \\
\hline PVAR & 990.02 & -923.65 & -1187.20 \\
CSV-PVAR & -917.47 & -887.59 & -1050.79 \\
CSVM-PVAR & -904.54 & $\mathbf{- 8 5 9 . 7 9}$ & -1051.38 \\
CSVM-PVAR-F & -921.48 & -875.77 & -1053.61 \\
CSVM-PVAR-R1 & $-\mathbf{9 0 3 . 6 4}$ & -869.16 & -1041.01 \\
CSVM-PVAR-R2 & -905.76 & -869.02 & -1047.93 \\
CSVM-PVAR-R3 & -917.23 & -879.18 & -1053.87 \\
CSVM-PVAR-R4 & -916.41 & -870.20 & $\mathbf{- 1 0 3 7 . 9 5}$ \\
\hline \hline
\end{tabular}

Note: Best model for each country is in bold. 
Appendix B.2. Uncertainty Measures

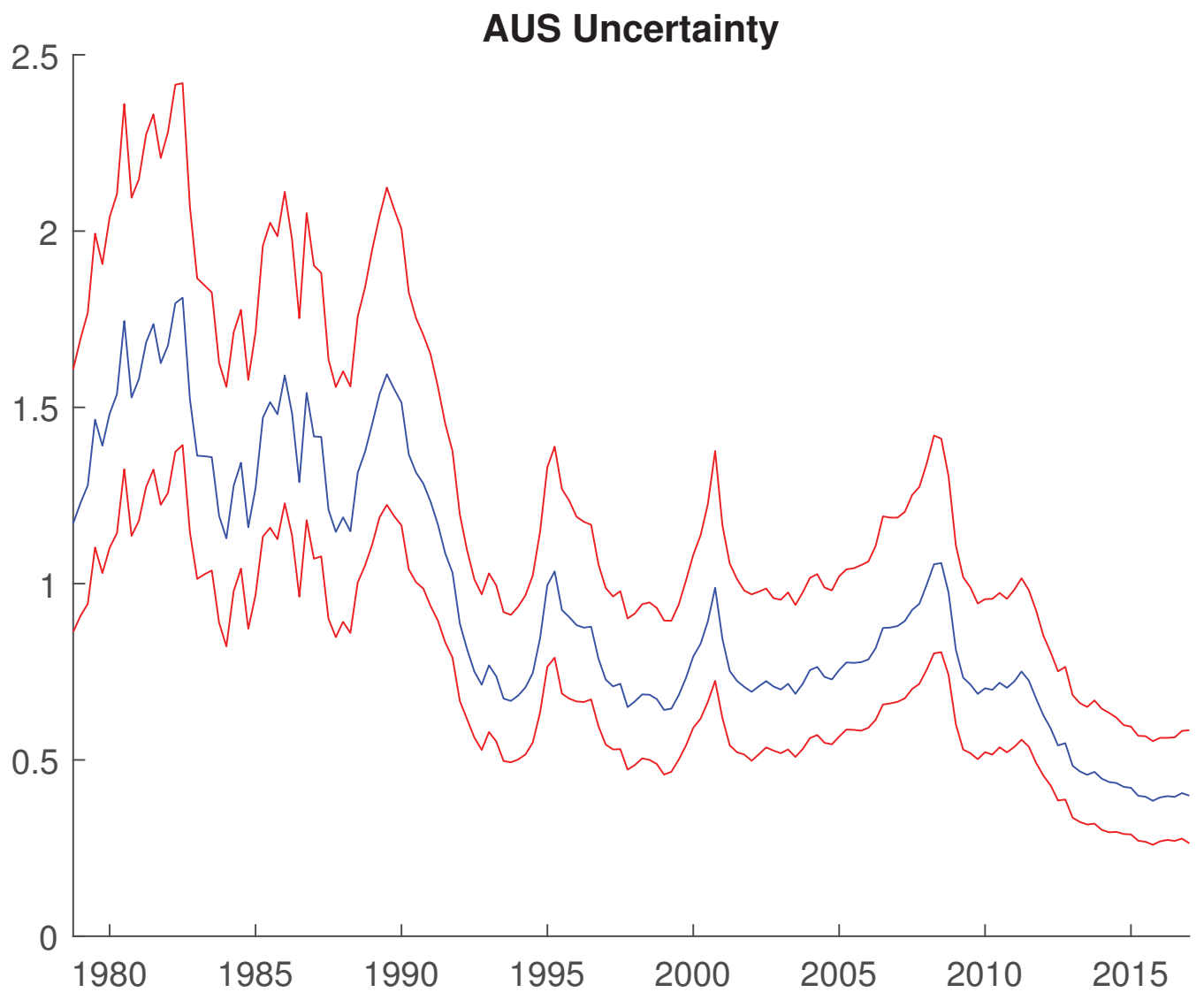

Figure B.1: Uncertainty Index: Australia

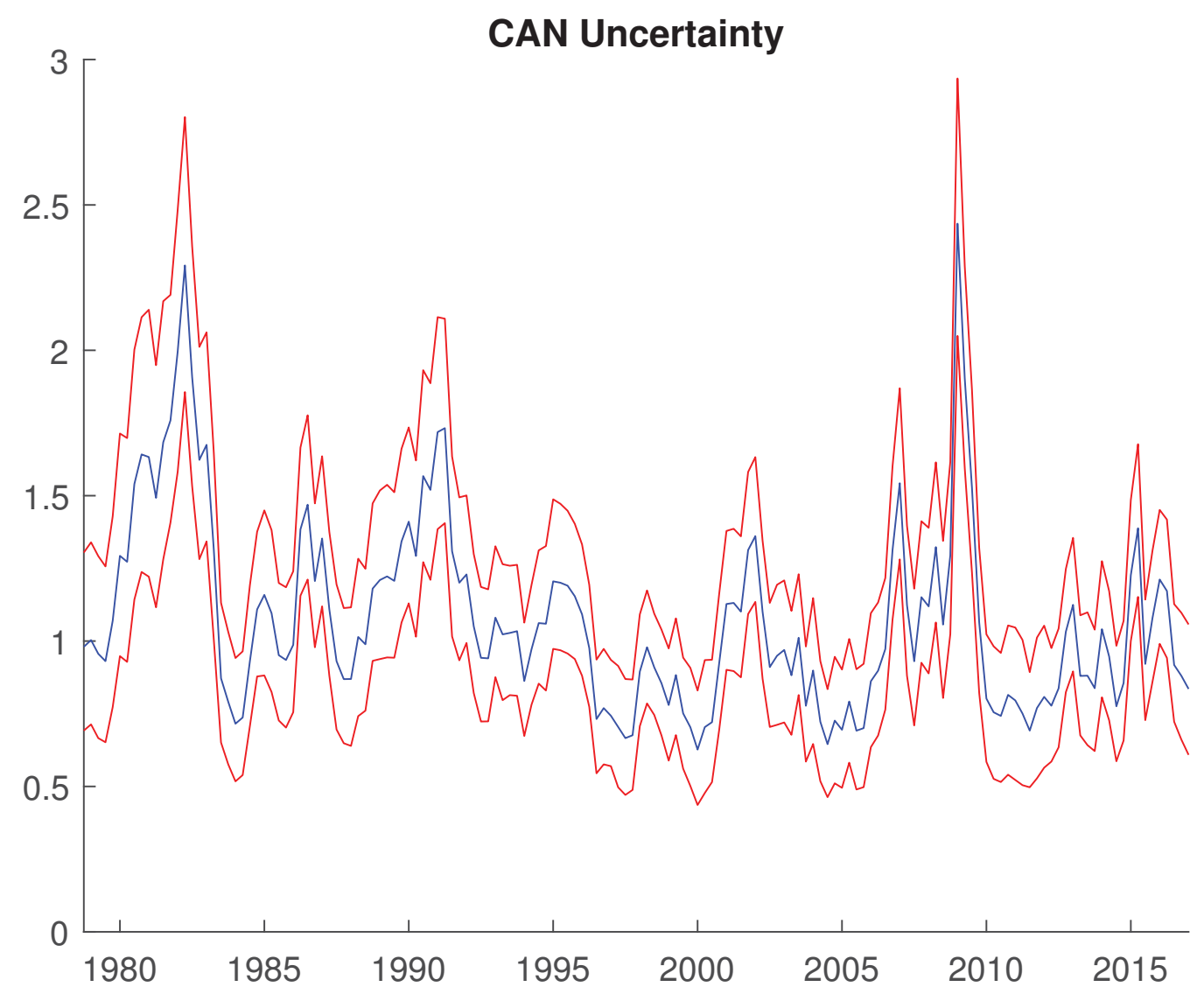

Figure B.2: Uncertainty Index: Canada 
Appendix B.3. Estimation Results

Appendix B.3.1. Australia

Table B.3: Estimated A matrix of CSVM-PVAR-R1 for Australia

\begin{tabular}{cl|cl}
\hline-0.93 & $(-1.84,-0.29)$ & 0.00 & $(0.00,0.00)$ \\
1.05 & $(0.42,2.00)$ & 0.00 & $(0.00,0.00)$ \\
0.03 & $(-0.12,0.20)$ & 0.00 & $(0.00,0.00)$ \\
\hline-0.32 & $(-1.07,0.30)$ & 0.52 & $(-1.80,2.76)$ \\
0.37 & $(-0.19,1.06)$ & 3.17 & $(1.18,5.28)$ \\
-0.54 & $(-1.06,-0.22)$ & 3.48 & $(2.08,5.32)$ \\
\hline
\end{tabular}
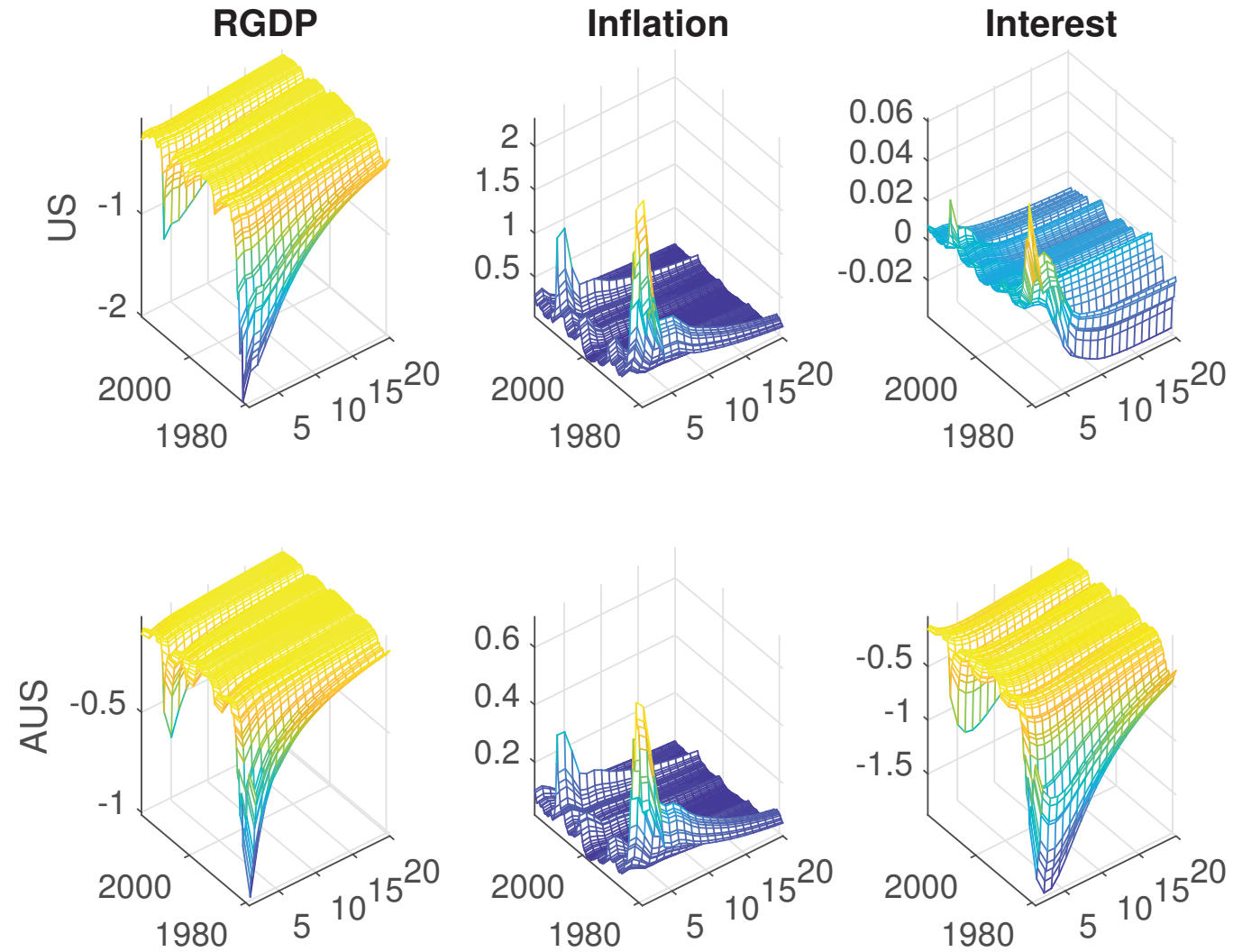

Figure B.3: GIRF: 1\% uncertainty shock from US 


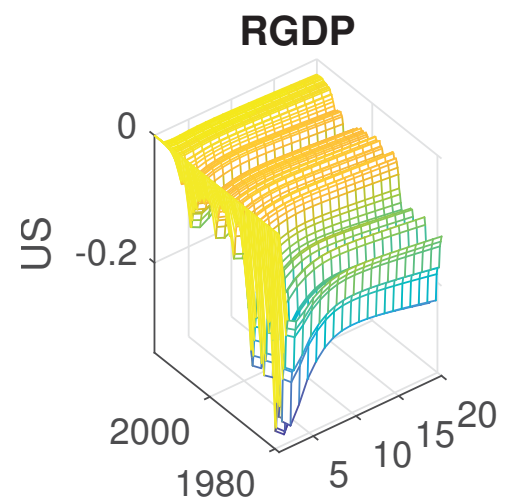

Inflation

Interest
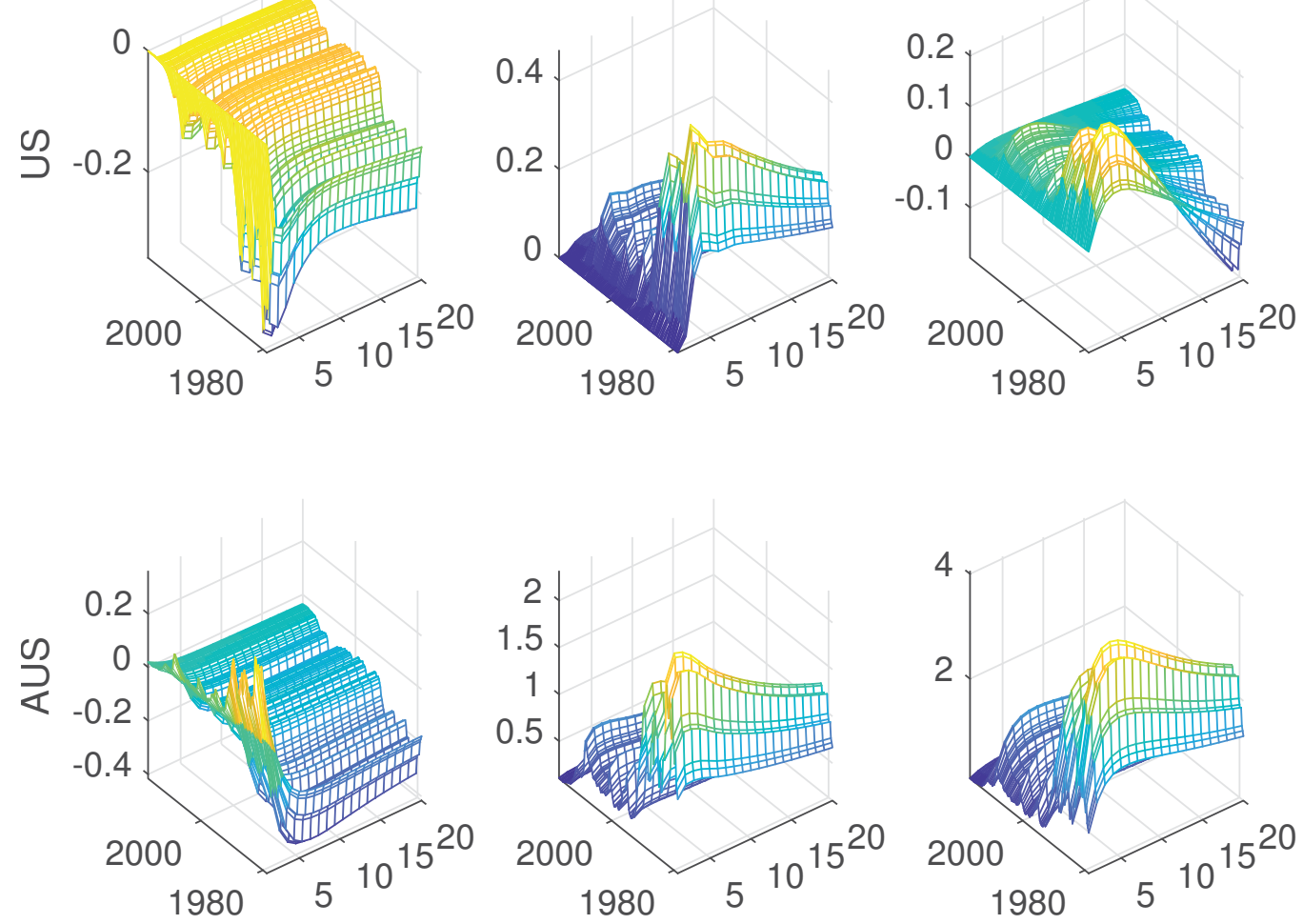

Figure B.4: GIRF: 1\% uncertainty shock from Australia

Appendix B.3.2. Canada

Table B.4: Estimated A matrix of CSVM-PVAR for Canada

\begin{tabular}{cl|cl}
-3.35 & $(-5.40,-1.65)$ & 1.59 & $(0.24,3.10)$ \\
1.13 & $(-0.43,2.71)$ & 2.30 & $(1.14,3.61)$ \\
-1.25 & $(-2.35,-0.45)$ & 1.93 & $(1.30,2.73)$ \\
\hline-2.39 & $(-4.08,-0.98)$ & 0.90 & $(-0.40,2.28)$ \\
1.64 & $(-0.02,3.44)$ & 1.78 & $(0.42,3.25)$ \\
-0.88 & $(-1.68,-0.31)$ & 1.56 & $(0.99,2.25)$ \\
\hline
\end{tabular}




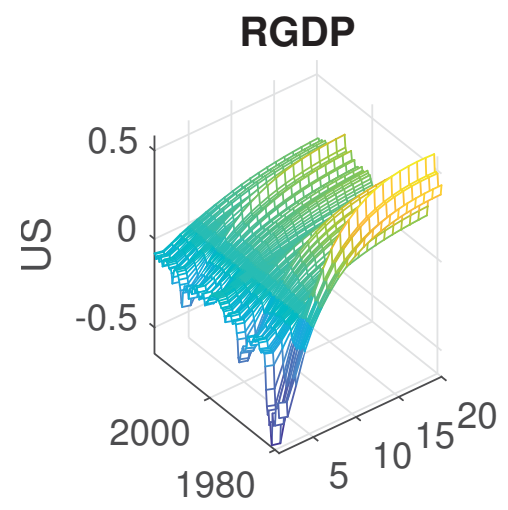

Inflation

Interest
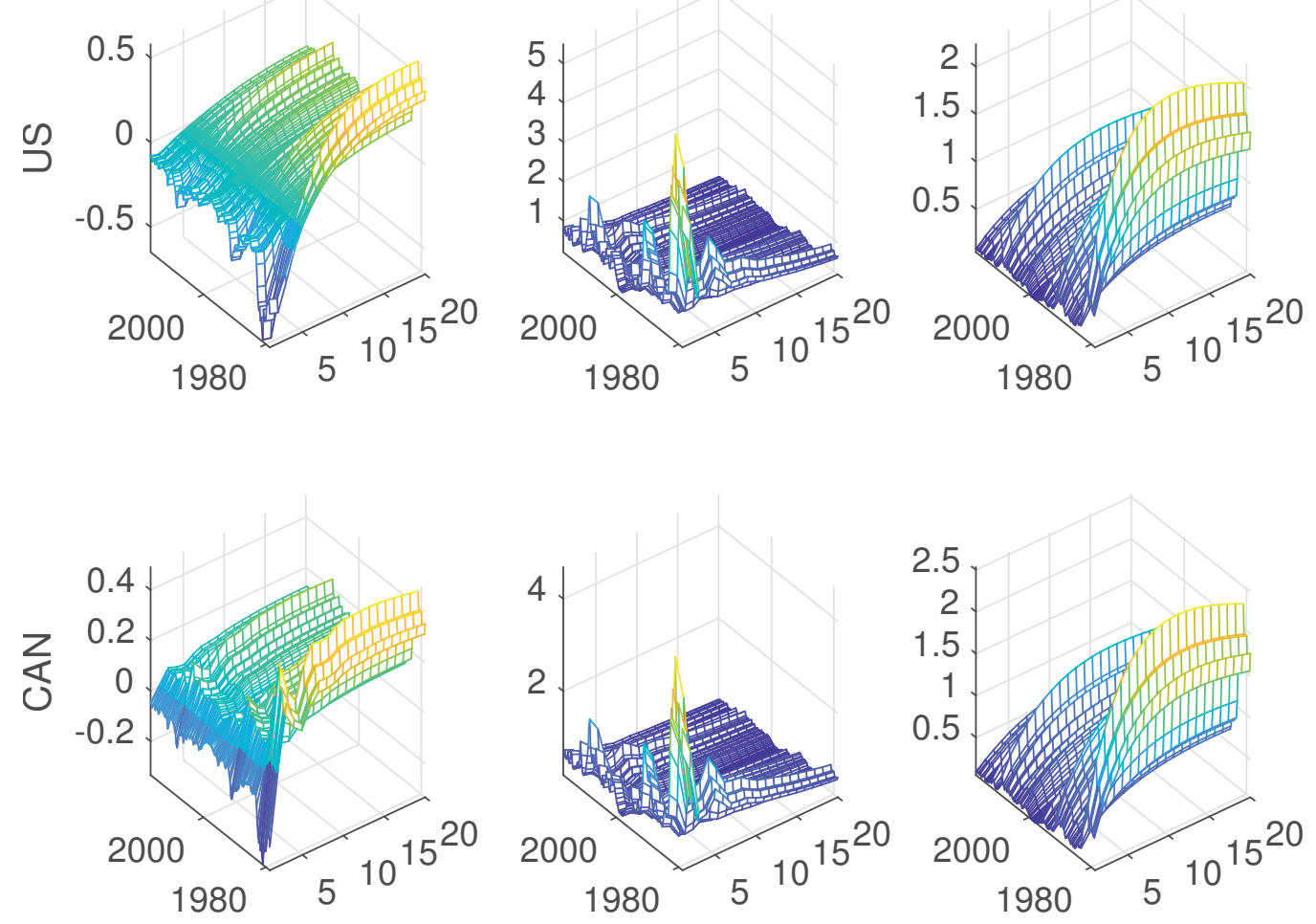

Figure B.5: GIRF: $1 \%$ uncertainty shock from US
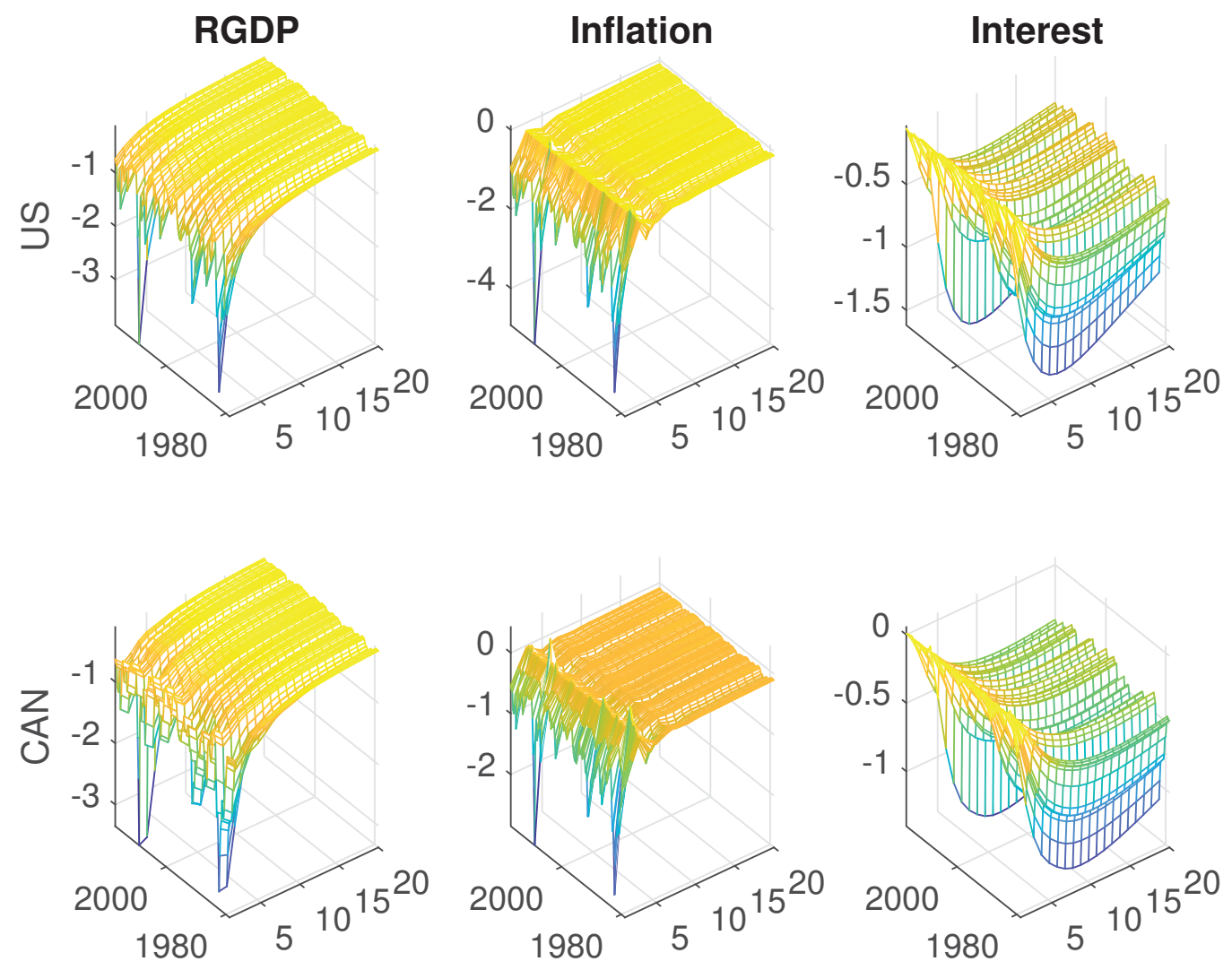

Figure B.6: GIRF: 1\% uncertainty shock from Canada 
Table B.5: Estimated A matrix of CSVM-PVAR-R4 for New Zealand

\begin{tabular}{cl|cc}
\hline-1.03 & $(-1.89,-0.35)$ & 0.00 & $(0.00,0.00)$ \\
1.36 & $(0.62,2.33)$ & 0.00 & $(0.00,0.00)$ \\
0.15 & $(-0.02,0.37)$ & 0.00 & $(0.00,0.00)$ \\
\hline-0.54 & $(-1.55,0.34)$ & 0.00 & $(0.00,0.00)$ \\
1.82 & $(0.91,2.99)$ & 0.00 & $(0.00,0.00)$ \\
-0.06 & $(-0.25,0.11)$ & 0.00 & $(0.00,0.00)$ \\
\hline
\end{tabular}
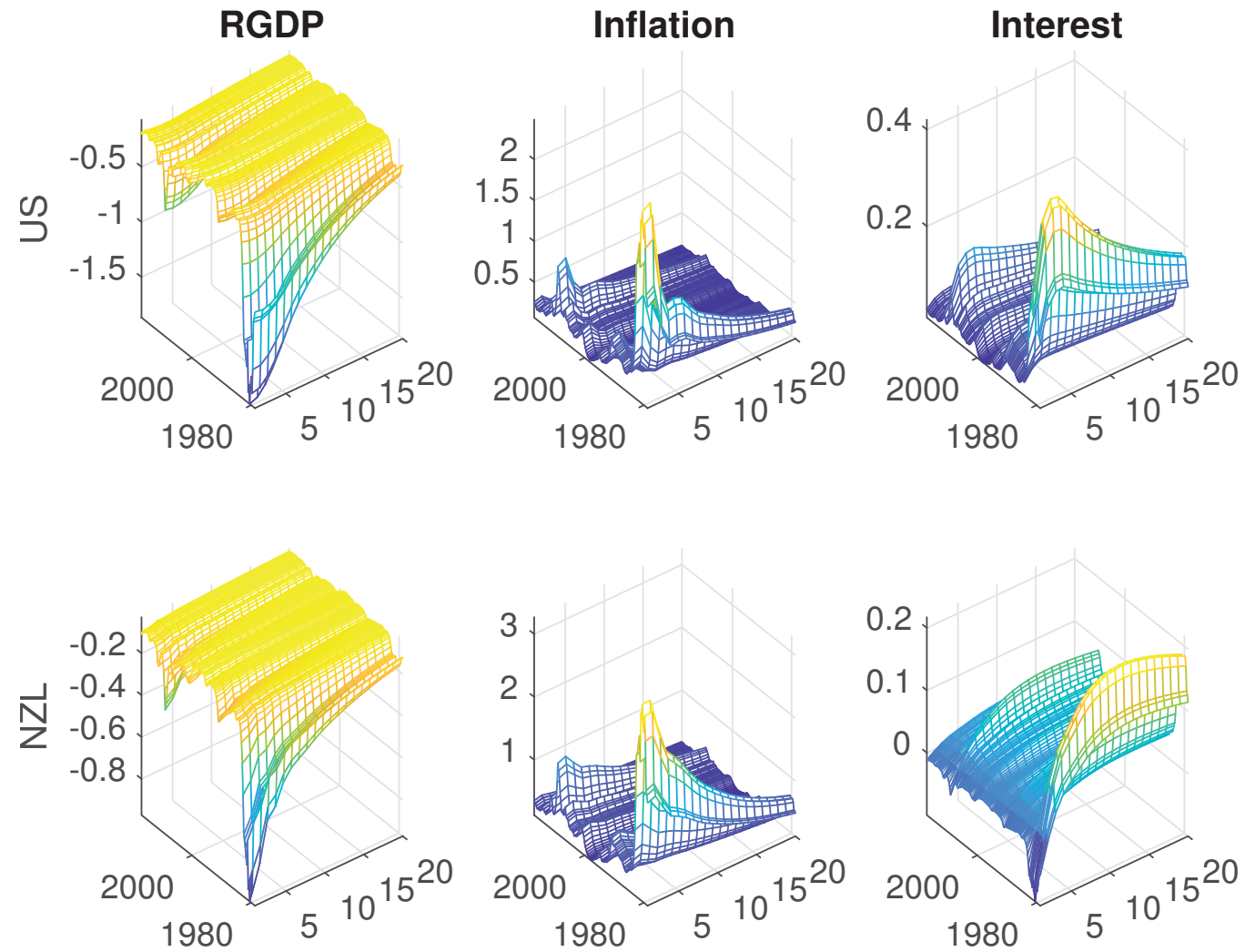

Figure B.7: GIRF: $1 \%$ uncertainty shock from US

Appendix B.4. Forecasting Results

Appendix B.4.1. Australia

Table B.6: Real GDP forecast for Australia

\begin{tabular}{|c|c|c|c|c|c|c|c|c|c|c|c|c|c|c|c|c|}
\hline & \multicolumn{4}{|c|}{ RMSFE } & \multicolumn{4}{|c|}{ MAE } & \multicolumn{4}{|c|}{ ALPS } & \multicolumn{4}{|c|}{ ACRPS } \\
\hline & $\mathrm{h}=1$ & $\mathrm{~h}=2$ & $\mathrm{~h}=4$ & $\mathrm{~h}=8$ & $\mathrm{~h}=1$ & $\mathrm{~h}=2$ & $\mathrm{~h}=4$ & $\mathrm{~h}=8$ & $\mathrm{~h}=1$ & $\mathrm{~h}=2$ & $\mathrm{~h}=4$ & $\mathrm{~h}=8$ & $\mathrm{~h}=1$ & $\mathrm{~h}=2$ & $\mathrm{~h}=4$ & $\mathrm{~h}=8$ \\
\hline PVAR & 1.00 & 1.00 & 1.00 & 1.00 & 1.00 & 1.00 & 1.00 & 1.00 & 0.00 & 0.00 & 0.00 & 0.00 & 1.00 & 1.00 & 1.00 & 1.00 \\
\hline CSV-PVAR & 0.99 & 0.97 & 0.96 & 0.98 & 0.97 & 0.96 & 0.95 & 0.98 & 0.05 & 0.08 & 0.12 & 0.16 & 0.97 & 0.95 & 0.93 & 0.93 \\
\hline CSVM-PVAR & 0.98 & 0.94 & 0.95 & 1.01 & 0.95 & 0.89 & 0.92 & 1.01 & 0.11 & 0.17 & 0.17 & 0.13 & 0.96 & 0.91 & 0.92 & 0.97 \\
\hline CSVM-PVAR-R1 & 0.99 & 0.95 & 0.94 & 0.97 & 0.96 & 0.92 & 0.92 & 0.97 & 0.10 & 0.15 & 0.17 & 0.15 & 0.97 & 0.92 & 0.91 & 0.93 \\
\hline CSVM-PVAR-R2 & 0.99 & 0.95 & 0.94 & 0.97 & 0.97 & 0.92 & 0.92 & 0.97 & 0.10 & 0.15 & 0.17 & 0.15 & 0.97 & 0.93 & 0.91 & 0.94 \\
\hline CSVM-PVAR-R3 & 0.98 & 0.96 & 0.94 & 0.98 & 0.96 & 0.94 & 0.92 & 0.97 & 0.10 & 0.14 & 0.16 & 0.16 & 0.96 & 0.93 & 0.91 & 0.93 \\
\hline CSVM-PVAR-R4 & 0.99 & 0.96 & 0.93 & 0.97 & 0.97 & 0.93 & 0.92 & 0.96 & 0.05 & 0.10 & 0.15 & 0.18 & 0.98 & 0.95 & 0.91 & 0.92 \\
\hline CSVM-PVAR-F & 0.99 & 0.96 & 0.94 & 0.98 & 0.98 & 0.94 & 0.92 & 0.97 & 0.09 & 0.15 & 0.17 & 0.17 & 0.99 & 0.95 & 0.92 & 0.94 \\
\hline
\end{tabular}


Table B.7: inflation forecast for Australia

\begin{tabular}{|c|c|c|c|c|c|c|c|c|c|c|c|c|c|c|c|c|}
\hline & \multicolumn{4}{|c|}{ RMSFE } & \multicolumn{4}{|c|}{ MAE } & \multicolumn{4}{|l|}{ ALPS } & \multicolumn{4}{|c|}{ ACRPS } \\
\hline & $\mathrm{h}=1$ & $\mathrm{~h}=2$ & $\mathrm{~h}=4$ & $\mathrm{~h}=8$ & $\mathrm{~h}=1$ & $\mathrm{~h}=2$ & $\mathrm{~h}=4$ & $\mathrm{~h}=8$ & $\mathrm{~h}=1$ & $\mathrm{~h}=2$ & $\mathrm{~h}=4$ & $\mathrm{~h}=8$ & $\mathrm{~h}=1$ & $\mathrm{~h}=2$ & $\mathrm{~h}=4$ & $\mathrm{~h}=8$ \\
\hline PVAR & 1.00 & 1.00 & 1.00 & 1.00 & 1.00 & 1.00 & 1.00 & 1.00 & 0.00 & 0.00 & 0.00 & 0.00 & 1.00 & 1.00 & 1.00 & 1.00 \\
\hline CSV-PVAR & 1.00 & 0.99 & 0.99 & 0.98 & 0.96 & 0.97 & 0.97 & 0.97 & -0.01 & 0.02 & 0.05 & 0.13 & 0.98 & 0.98 & 0.97 & 0.95 \\
\hline CSVM-PVAR & 1.00 & 0.99 & 0.97 & 0.98 & 0.95 & 0.93 & 0.91 & 0.96 & 0.10 & 0.14 & 0.16 & 0.20 & 0.97 & 0.95 & 0.93 & 0.92 \\
\hline CSVM-PVAR-R1 & 0.97 & 0.95 & 0.93 & 0.94 & 0.94 & 0.91 & 0.88 & 0.93 & 0.12 & 0.15 & 0.20 & 0.23 & 0.95 & 0.93 & 0.89 & 0.88 \\
\hline CSVM-PVAR-R2 & 0.97 & 0.95 & 0.92 & 0.93 & 0.94 & 0.91 & 0.87 & 0.92 & 0.13 & 0.15 & 0.20 & 0.23 & 0.95 & 0.93 & 0.89 & 0.88 \\
\hline CSVM-PVAR-R3 & 0.97 & 0.95 & 0.92 & 0.93 & 0.95 & 0.92 & 0.89 & 0.93 & 0.11 & 0.16 & 0.20 & 0.22 & 0.95 & 0.92 & 0.89 & 0.88 \\
\hline CSVM-PVAR-R4 & 1.00 & 0.99 & 0.98 & 0.99 & 0.95 & 0.94 & 0.91 & 0.96 & -0.01 & 0.00 & 0.09 & 0.14 & 0.98 & 0.98 & 0.95 & 0.94 \\
\hline CSVM-PVAR-F & 0.99 & 0.99 & 0.97 & 0.98 & 0.96 & 0.96 & 0.91 & 0.97 & 0.11 & 0.10 & 0.14 & 0.18 & 0.98 & 0.98 & 0.96 & 0.95 \\
\hline
\end{tabular}

Table B.8: Interest rate forecast for Australia

\begin{tabular}{|c|c|c|c|c|c|c|c|c|c|c|c|c|c|c|c|c|}
\hline & \multicolumn{4}{|c|}{ RMSFE } & \multicolumn{4}{|c|}{ MAE } & \multicolumn{4}{|c|}{ ALPS } & \multicolumn{4}{|c|}{ ACRPS } \\
\hline & $\mathrm{h}=1$ & $\mathrm{~h}=2$ & $\mathrm{~h}=4$ & $\mathrm{~h}=8$ & $\mathrm{~h}=1$ & $\mathrm{~h}=2$ & $\mathrm{~h}=4$ & $\mathrm{~h}=8$ & $\mathrm{~h}=1$ & $\mathrm{~h}=2$ & $\mathrm{~h}=4$ & $\mathrm{~h}=8$ & $\mathrm{~h}=1$ & $\mathrm{~h}=2$ & $\mathrm{~h}=4$ & $\mathrm{~h}=8$ \\
\hline PVAR & 1.00 & 1.00 & 1.00 & 1.00 & 1.00 & 1.00 & 1.00 & 1.00 & 0.00 & 0.00 & 0.00 & 0.00 & 1.00 & 1.00 & 1.00 & 1.00 \\
\hline CSV-PVAR & 0.96 & 0.99 & 1.01 & 1.00 & 0.88 & 0.91 & 0.98 & 1.00 & 0.43 & 0.31 & 0.19 & 0.16 & 0.82 & 0.88 & 0.94 & 0.96 \\
\hline CSVM-PVAR & 0.84 & 0.88 & 0.91 & 0.91 & 0.75 & 0.76 & 0.80 & 0.85 & 0.51 & 0.34 & 0.26 & 0.32 & 0.78 & 0.83 & 0.85 & 0.85 \\
\hline CSVM-PVAR-R1 & 0.77 & 0.81 & 0.82 & 0.82 & 0.71 & 0.71 & 0.76 & 0.81 & 0.50 & 0.37 & 0.27 & 0.40 & 0.79 & 0.81 & 0.79 & 0.76 \\
\hline CSVM-PVAR-R2 & 0.78 & 0.81 & 0.82 & 0.82 & 0.71 & 0.71 & 0.77 & 0.80 & 0.49 & 0.34 & 0.26 & 0.39 & 0.80 & 0.82 & 0.80 & 0.77 \\
\hline CSVM-PVAR-R3 & 0.83 & 0.85 & 0.83 & 0.83 & 0.75 & 0.74 & 0.78 & 0.81 & 0.44 & 0.23 & 0.21 & 0.29 & 0.83 & 0.85 & 0.81 & 0.78 \\
\hline CSVM-PVAR-R4 & 0.95 & 0.98 & 0.99 & 0.98 & 0.85 & 0.88 & 0.93 & 0.96 & 0.44 & 0.30 & 0.20 & 0.19 & 0.81 & 0.87 & 0.93 & 0.95 \\
\hline CSVM-PVAR-F & 0.97 & 1.00 & 1.01 & 0.99 & 0.93 & 0.95 & 1.00 & 1.00 & 0.26 & 0.18 & 0.13 & 0.19 & 0.92 & 0.97 & 1.02 & 1.00 \\
\hline
\end{tabular}

Appendix B.4.2. Canada

Table B.9: Real GDP forecast for Canada

\begin{tabular}{|c|c|c|c|c|c|c|c|c|c|c|c|c|c|c|c|c|}
\hline & \multicolumn{4}{|c|}{ RMSFE } & \multicolumn{4}{|c|}{ MAE } & \multicolumn{4}{|c|}{ ALPS } & \multicolumn{4}{|c|}{ ACRPS } \\
\hline & $\mathrm{h}=1$ & $\mathrm{~h}=2$ & $\mathrm{~h}=4$ & $\mathrm{~h}=8$ & $\mathrm{~h}=1$ & $\mathrm{~h}=2$ & $\mathrm{~h}=4$ & $\mathrm{~h}=8$ & $\mathrm{~h}=1$ & $\mathrm{~h}=2$ & $\mathrm{~h}=4$ & $\mathrm{~h}=8$ & $\mathrm{~h}=1$ & $\mathrm{~h}=2$ & $\mathrm{~h}=4$ & $\mathrm{~h}=8$ \\
\hline PVAR & 1.00 & 1.00 & 1.00 & 1.00 & 1.00 & 1.00 & 1.00 & 1.00 & 0.00 & 0.00 & 0.00 & 0.00 & 1.00 & 1.00 & 1.00 & 1.00 \\
\hline CSV-PVAR & 0.96 & 0.94 & 0.97 & 1.02 & 0.96 & 0.95 & 0.97 & 1.02 & 0.04 & 0.09 & 0.06 & 0.03 & 0.97 & 0.95 & 0.98 & 1.03 \\
\hline CSVM-PVAR & 0.93 & 0.90 & 0.94 & 0.97 & 0.95 & 0.91 & 0.94 & 0.97 & 0.11 & 0.16 & 0.10 & 0.10 & 0.96 & 0.92 & 0.97 & 0.98 \\
\hline CSVM-PVAR-R1 & 0.95 & 0.92 & 0.94 & 0.95 & 0.96 & 0.95 & 0.94 & 0.96 & 0.06 & 0.09 & 0.08 & 0.09 & 0.98 & 0.94 & 0.96 & 0.97 \\
\hline CSVM-PVAR-R2 & 0.95 & 0.92 & 0.94 & 0.95 & 0.96 & 0.95 & 0.94 & 0.95 & 0.06 & 0.09 & 0.08 & 0.10 & 0.97 & 0.94 & 0.96 & 0.97 \\
\hline CSVM-PVAR-R3 & 0.96 & 0.92 & 0.95 & 0.97 & 0.95 & 0.93 & 0.95 & 0.97 & 0.09 & 0.14 & 0.09 & 0.10 & 0.96 & 0.93 & 0.97 & 0.98 \\
\hline CSVM-PVAR-R4 & 0.95 & 0.93 & 0.95 & 0.97 & 0.96 & 0.95 & 0.96 & 0.96 & 0.05 & 0.10 & 0.09 & 0.08 & 0.97 & 0.94 & 0.96 & 0.98 \\
\hline CSVM-PVAR-F & 0.92 & 0.90 & 0.93 & 0.95 & 0.95 & 0.92 & 0.93 & 0.96 & 0.09 & 0.14 & 0.12 & 0.11 & 0.95 & 0.93 & 0.96 & 0.97 \\
\hline
\end{tabular}

Table B.10: Inflation forecast for Canada

\begin{tabular}{|c|c|c|c|c|c|c|c|c|c|c|c|c|c|c|c|c|}
\hline & \multicolumn{4}{|c|}{ RMSFE } & \multicolumn{4}{|l|}{ MAE } & \multicolumn{4}{|c|}{ ALPS } & \multicolumn{4}{|c|}{ ACRPS } \\
\hline & $\mathrm{h}=1$ & $\mathrm{~h}=2$ & $\mathrm{~h}=4$ & $\mathrm{~h}=8$ & $\mathrm{~h}=1$ & $\mathrm{~h}=2$ & $\mathrm{~h}=4$ & $\mathrm{~h}=8$ & $\mathrm{~h}=1$ & $\mathrm{~h}=2$ & $\mathrm{~h}=4$ & $\mathrm{~h}=8$ & $\mathrm{~h}=1$ & $\mathrm{~h}=2$ & $\mathrm{~h}=4$ & $\mathrm{~h}=8$ \\
\hline PVAR & 1.00 & 1.00 & 1.00 & 1.00 & 1.00 & 1.00 & 1.00 & 1.00 & 0.00 & 0.00 & 0.00 & 0.00 & 1.00 & 1.00 & 1.00 & 1.00 \\
\hline CSV-PVAR & 0.97 & 0.94 & 0.97 & 0.92 & 0.96 & 0.94 & 0.95 & 0.92 & 0.02 & 0.04 & 0.01 & 0.09 & 0.98 & 0.95 & 0.96 & 0.90 \\
\hline CSVM-PVAR & 0.94 & 0.94 & 0.95 & 0.89 & 0.91 & 0.91 & 0.86 & 0.86 & 0.11 & 0.07 & 0.06 & 0.11 & 0.94 & 0.93 & 0.91 & 0.84 \\
\hline CSVM-PVAR-R1 & 0.95 & 0.93 & 0.93 & 0.88 & 0.92 & 0.90 & 0.86 & 0.87 & 0.04 & 0.08 & 0.08 & 0.15 & 0.96 & 0.93 & 0.90 & 0.83 \\
\hline CSVM-PVAR-R2 & 0.95 & 0.93 & 0.93 & 0.89 & 0.92 & 0.90 & 0.86 & 0.87 & 0.04 & 0.08 & 0.07 & 0.15 & 0.96 & 0.93 & 0.91 & 0.84 \\
\hline CSVM-PVAR-R3 & 0.95 & 0.91 & 0.94 & 0.89 & 0.93 & 0.88 & 0.88 & 0.86 & 0.00 & 0.08 & 0.04 & 0.10 & 0.98 & 0.92 & 0.92 & 0.84 \\
\hline CSVM-PVAR-R4 & 0.95 & 0.92 & 0.93 & 0.89 & 0.91 & 0.90 & 0.87 & 0.87 & 0.07 & 0.09 & 0.05 & 0.13 & 0.96 & 0.93 & 0.92 & 0.84 \\
\hline CSVM-PVAR-F & 0.93 & 0.91 & 0.93 & 0.91 & 0.90 & 0.89 & 0.87 & 0.88 & 0.14 & 0.13 & 0.04 & 0.09 & 0.93 & 0.91 & 0.92 & 0.86 \\
\hline
\end{tabular}

Table B.11: Interest rate forecast for Canada

\begin{tabular}{|c|c|c|c|c|c|c|c|c|c|c|c|c|c|c|c|c|}
\hline & \multicolumn{4}{|c|}{ RMSFE } & \multicolumn{4}{|c|}{ MAE } & \multicolumn{4}{|c|}{ ALPS } & \multicolumn{4}{|c|}{ ACRPS } \\
\hline & $\mathrm{h}=1$ & $\mathrm{~h}=2$ & $\mathrm{~h}=4$ & $\mathrm{~h}=8$ & $\mathrm{~h}=1$ & $\mathrm{~h}=2$ & $\mathrm{~h}=4$ & $\mathrm{~h}=8$ & $\mathrm{~h}=1$ & $\mathrm{~h}=2$ & $\mathrm{~h}=4$ & $\mathrm{~h}=8$ & $\mathrm{~h}=1$ & $\mathrm{~h}=2$ & $\mathrm{~h}=4$ & $\mathrm{~h}=8$ \\
\hline PVAR & 1.00 & 1.00 & 1.00 & 1.00 & 1.00 & 1.00 & 1.00 & 1.00 & 0.00 & 0.00 & 0.00 & 0.00 & 1.00 & 1.00 & 1.00 & 1.00 \\
\hline CSV-PVAR & 1.01 & 0.92 & 0.84 & 0.80 & 0.99 & 0.93 & 0.80 & 0.74 & 0.21 & 0.25 & 0.23 & 0.23 & 0.94 & 0.87 & 0.81 & 0.77 \\
\hline CSVM-PVAR & 0.93 & 0.87 & 0.82 & 0.81 & 0.91 & 0.89 & 0.74 & 0.67 & 0.24 & 0.28 & 0.29 & 0.30 & 0.91 & 0.87 & 0.80 & 0.74 \\
\hline CSVM-PVAR-R1 & 0.95 & 0.94 & 0.92 & 0.89 & 0.91 & 0.95 & 0.88 & 0.78 & 0.29 & 0.25 & 0.15 & 0.04 & 0.93 & 0.93 & 0.88 & 0.81 \\
\hline CSVM-PVAR-R2 & 0.95 & 0.94 & 0.92 & 0.89 & 0.90 & 0.95 & 0.87 & 0.79 & 0.29 & 0.25 & 0.15 & 0.04 & 0.93 & 0.92 & 0.88 & 0.81 \\
\hline CSVM-PVAR-R3 & 0.98 & 0.94 & 0.92 & 0.88 & 0.91 & 0.94 & 0.85 & 0.78 & 0.26 & 0.26 & 0.17 & 0.04 & 0.95 & 0.92 & 0.88 & 0.81 \\
\hline CSVM-PVAR-R4 & 0.94 & 0.90 & 0.86 & 0.85 & 0.93 & 0.92 & 0.80 & 0.73 & 0.27 & 0.28 & 0.24 & 0.18 & 0.90 & 0.87 & 0.82 & 0.77 \\
\hline CSVM-PVAR-F & 0.99 & 0.95 & 0.92 & 0.91 & 0.95 & 0.96 & 0.90 & 0.88 & 0.14 & 0.16 & 0.17 & 0.15 & 1.00 & 0.97 & 0.92 & 0.89 \\
\hline
\end{tabular}


Table B.12: Real GDP forecast for New Zealand

\begin{tabular}{|c|c|c|c|c|c|c|c|c|c|c|c|c|c|c|c|c|}
\hline & \multicolumn{4}{|c|}{ RMSFE } & \multicolumn{4}{|c|}{ MAE } & \multicolumn{4}{|c|}{ ALPS } & \multicolumn{4}{|c|}{ ACRPS } \\
\hline & $\mathrm{h}=1$ & $\mathrm{~h}=2$ & $\mathrm{~h}=4$ & $\mathrm{~h}=8$ & $\mathrm{~h}=1$ & $\mathrm{~h}=2$ & $\mathrm{~h}=4$ & $\mathrm{~h}=8$ & $\mathrm{~h}=1$ & $\mathrm{~h}=2$ & $\mathrm{~h}=4$ & $\mathrm{~h}=8$ & $\mathrm{~h}=1$ & $\mathrm{~h}=2$ & $\mathrm{~h}=4$ & $\mathrm{~h}=8$ \\
\hline PVAR & 1.00 & 1.00 & 1.00 & 1.00 & 1.00 & 1.00 & 1.00 & 1.00 & 0.00 & 0.00 & 0.00 & 0.00 & 1.00 & 1.00 & 1.00 & 1.00 \\
\hline CSV-PVAR & 0.98 & 1.02 & 1.02 & 1.02 & 0.98 & 1.02 & 1.00 & 1.02 & 0.24 & 0.25 & 0.31 & 0.30 & 0.91 & 0.92 & 0.88 & 0.88 \\
\hline CSVM-PVAR & 0.98 & 1.02 & 1.01 & 1.01 & 0.98 & 1.02 & 1.01 & 1.00 & 0.27 & 0.27 & 0.29 & 0.29 & 0.90 & 0.91 & 0.89 & 0.88 \\
\hline CSVM-PVAR-R 1 & 0.98 & 1.02 & 1.01 & 1.01 & 0.98 & 1.02 & 1.01 & 1.01 & 0.27 & 0.28 & 0.31 & 0.31 & 0.90 & 0.91 & 0.88 & 0.87 \\
\hline CSVM-PVAR-R2 & 0.98 & 1.02 & 1.01 & 1.01 & 0.98 & 1.02 & 1.01 & 1.01 & 0.27 & 0.28 & 0.31 & 0.31 & 0.90 & 0.91 & 0.88 & 0.87 \\
\hline CSVM-PVAR-R3 & 0.98 & 1.01 & 1.00 & 1.01 & 0.98 & 1.02 & 1.00 & 1.01 & 0.25 & 0.26 & 0.31 & 0.31 & 0.91 & 0.91 & 0.88 & 0.87 \\
\hline CSVM-PVAR-R4 & 0.98 & 1.02 & 1.02 & 1.02 & 0.98 & 1.02 & 1.00 & 1.02 & 0.24 & 0.24 & 0.31 & 0.31 & 0.91 & 0.92 & 0.89 & 0.88 \\
\hline CSVM-PVAR-F & 0.97 & 1.02 & 1.01 & 1.01 & 0.97 & 1.02 & 1.01 & 1.01 & 0.27 & 0.26 & 0.30 & 0.31 & 0.90 & 0.91 & 0.88 & 0.87 \\
\hline
\end{tabular}

Table B.13: Inflation forecast for New Zealand

\begin{tabular}{|c|c|c|c|c|c|c|c|c|c|c|c|c|c|c|c|c|}
\hline & \multicolumn{4}{|c|}{ RMSFE } & \multicolumn{4}{|l|}{ MAE } & \multicolumn{4}{|c|}{ ALPS } & \multicolumn{4}{|c|}{ ACRPS } \\
\hline & $\mathrm{h}=1$ & $\mathrm{~h}=2$ & $\mathrm{~h}=4$ & $\mathrm{~h}=8$ & $\mathrm{~h}=1$ & $\mathrm{~h}=2$ & $\mathrm{~h}=4$ & $\mathrm{~h}=8$ & $\mathrm{~h}=1$ & $\mathrm{~h}=2$ & $\mathrm{~h}=4$ & $\mathrm{~h}=8$ & $\mathrm{~h}=1$ & $\mathrm{~h}=2$ & $\mathrm{~h}=4$ & $\mathrm{~h}=8$ \\
\hline PVAR & 1.00 & 1.00 & 1.00 & 1.00 & 1.00 & 1.00 & 1.00 & 1.00 & 0.00 & 0.00 & 0.00 & 0.00 & 1.00 & 1.00 & 1.00 & 1.00 \\
\hline CSV-PVAR & 0.93 & 0.93 & 0.86 & 0.82 & 0.93 & 0.89 & 0.82 & 0.81 & 0.25 & 0.25 & 0.34 & 0.39 & 0.87 & 0.86 & 0.81 & 0.76 \\
\hline CSVM-PVAR & 0.84 & 0.83 & 0.80 & 0.72 & 0.84 & 0.77 & 0.71 & 0.67 & 0.36 & 0.38 & 0.44 & 0.51 & 0.81 & 0.78 & 0.72 & 0.63 \\
\hline CSVM-PVAR-R1 & 0.84 & 0.84 & 0.79 & 0.71 & 0.85 & 0.76 & 0.70 & 0.65 & 0.36 & 0.38 & 0.45 & 0.53 & 0.81 & 0.78 & 0.71 & 0.62 \\
\hline CSVM-PVAR-R2 & 0.84 & 0.84 & 0.79 & 0.71 & 0.85 & 0.77 & 0.70 & 0.65 & 0.36 & 0.38 & 0.44 & 0.53 & 0.81 & 0.78 & 0.72 & 0.62 \\
\hline CSVM-PVAR-R3 & 0.89 & 0.83 & 0.74 & 0.71 & 0.92 & 0.79 & 0.68 & 0.65 & 0.29 & 0.35 & 0.48 & 0.54 & 0.86 & 0.80 & 0.71 & 0.64 \\
\hline CSVM-PVAR-R4 & 0.85 & 0.85 & 0.81 & 0.75 & 0.84 & 0.78 & 0.74 & 0.71 & 0.33 & 0.35 & 0.42 & 0.48 & 0.82 & 0.80 & 0.75 & 0.68 \\
\hline CSVM-PVAR-F & 0.84 & 0.84 & 0.78 & 0.71 & 0.83 & 0.79 & 0.71 & 0.66 & 0.34 & 0.36 & 0.46 & 0.53 & 0.82 & 0.81 & 0.73 & 0.64 \\
\hline
\end{tabular}

Table B.14: Interest rate forecast for New Zealand

\begin{tabular}{|c|c|c|c|c|c|c|c|c|c|c|c|c|c|c|c|c|}
\hline & \multicolumn{4}{|c|}{ RMSFE } & \multicolumn{4}{|c|}{ MAE } & \multicolumn{4}{|c|}{ ALPS } & \multicolumn{4}{|c|}{ ACRPS } \\
\hline & $\mathrm{h}=1$ & $\mathrm{~h}=2$ & $\mathrm{~h}=4$ & $\mathrm{~h}=8$ & $\mathrm{~h}=1$ & $\mathrm{~h}=2$ & $\mathrm{~h}=4$ & $\mathrm{~h}=8$ & $\mathrm{~h}=1$ & $\mathrm{~h}=2$ & $\mathrm{~h}=4$ & $\mathrm{~h}=8$ & $\mathrm{~h}=1$ & $\mathrm{~h}=2$ & $\mathrm{~h}=4$ & $\mathrm{~h}=8$ \\
\hline PVAR & 1.00 & 1.00 & 1.00 & 1.00 & 1.00 & 1.00 & 1.00 & 1.00 & 0.00 & 0.00 & 0.00 & 0.00 & 1.00 & 1.00 & 1.00 & 1.00 \\
\hline CSV-PVAR & 0.95 & 0.95 & 0.93 & 0.91 & 0.92 & 0.95 & 0.94 & 0.95 & 0.36 & 0.29 & 0.18 & 0.10 & 0.85 & 0.89 & 0.90 & 0.90 \\
\hline CSVM-PVAR & 0.92 & 0.92 & 0.89 & 0.85 & 0.96 & 0.97 & 0.94 & 0.86 & 0.32 & 0.19 & 0.03 & -0.02 & 0.88 & 0.91 & 0.88 & 0.81 \\
\hline CSVM-PVAR-R1 & 0.93 & 0.93 & 0.89 & 0.85 & 0.97 & 0.99 & 0.94 & 0.85 & 0.32 & 0.18 & 0.01 & -0.05 & 0.88 & 0.92 & 0.88 & 0.81 \\
\hline CSVM-PVAR-R2 & 0.93 & 0.93 & 0.89 & 0.85 & 0.97 & 0.99 & 0.95 & 0.85 & 0.32 & 0.19 & 0.02 & -0.06 & 0.88 & 0.92 & 0.89 & 0.81 \\
\hline CSVM-PVAR-R3 & 0.90 & 0.89 & 0.84 & 0.82 & 0.92 & 0.94 & 0.89 & 0.85 & 0.33 & 0.23 & 0.12 & 0.07 & 0.86 & 0.88 & 0.85 & 0.81 \\
\hline CSVM-PVAR-R4 & 0.94 & 0.95 & 0.93 & 0.90 & 0.92 & 0.95 & 0.93 & 0.95 & 0.37 & 0.30 & 0.19 & 0.07 & 0.85 & 0.89 & 0.90 & 0.90 \\
\hline CSVM-PVAR-F & 0.94 & 0.95 & 0.90 & 0.86 & 0.96 & 0.98 & 0.95 & 0.89 & 0.28 & 0.21 & 0.12 & 0.06 & 0.90 & 0.93 & 0.91 & 0.85 \\
\hline
\end{tabular}

\section{References}

Baker, Scott R, Nicholas Bloom, and Steven J Davis, "Measuring economic policy uncertainty," The Quarterly Journal of Economics, 2016, 131 (4), 1593-1636.

Basu, Susanto and Brent Bundick, "Uncertainty shocks in a model of effective demand," Econometrica, 2017, $85(3), 937-958$.

Bloom, Nicholas, "The impact of uncertainty shocks," econometrica, 2009, r77 (3), 623-685.

Born, Benjamin and Johannes Pfeifer, "Policy risk and the business cycle," Journal of Monetary Economics, 2014, 68, 68-85.

Caggiano, Giovanni, Efrem Castelnuovo, and Juan Manuel Figueres, "Economic policy uncertainty spillovers in booms and busts," Working paper, 2017.

Canova, Fabio, "The transmission of US shocks to Latin America," Journal of Applied econometrics, 2005, 20 (2), 229-251.

_ and Matteo Ciccarelli, "ClubMed? Cyclical fluctuations in the Mediterranean basin," Journal of International Economics, 2012, 88 (1), 162-175.

_ and _, "Panel Vector Autoregressive Models: A Survey.," in "VAR Models in Macroeconomics-New Developments and Applications: Essays in Honor of Christopher A. Sims," Emerald Group Publishing Limited, 2013, pp. 205-246. 
_ , _ and Eva Ortega, "Similarities and convergence in G-7 cycles," Journal of Monetary economics, 2007, $54(3), 850-878$.

Carriero, Andrea, Haroon Mumtaz, Konstantinos Theodoridis, and Angeliki Theophilopoulou, "The impact of uncertainty shocks under measurement error: A proxy SVAR approach," Journal of Money, Credit and Banking, 2015, 47 (6), 1223-1238.

_ , Todd E Clark, and Massimiliano Marcellino, "Common drifting volatility in large Bayesian VARs," Journal of Business \& Economic Statistics, 2016, 34 (3), 375-390.

_ , Todd E. Clark, and Massimiliano Marcellino, "Measuring Uncertainty and Its Impact on the Economy," The Review of Economics and Statistics, 2017, 0 (forthcoming), null.

Chan, Joshua CC, "The stochastic volatility in mean model with time-varying parameters: An application to inflation modeling," Journal of Business 85 Economic Statistics, 2017, 35 (1), 17-28.

_ and Angelia L Grant, "On the observed-data deviance information criterion for volatility modeling," Journal of Financial Econometrics, 2016, 14 (4), 772-802.

_ and Ivan Jeliazkov, "Efficient simulation and integrated likelihood estimation in state space models," International Journal of Mathematical Modelling and Numerical Optimisation, 2009,1 (1-2), 101-120.

Cross, Jamie, Joshua CC Chan, Timothy Kam, and Aubrey Poon, "Economic, Fiscal- or Monetarypolicy Uncertainty Shocks: What Matters for a Small Open Economy?," Working paper, 2017.

Del Negro, Marco and Giorgio E Primiceri, "Time varying structural vector autoregressions and monetary policy: a corrigendum," The review of economic studies, 2015, 82 (4), 1342-1345.

Faccini, Renato, Haroon Mumtaz, and Paolo Surico, "International fiscal spillovers," Journal of International Economics, 2016, 99, 31-45.

Fernández-Villaverde, Jesús, Pablo Guerrón-Quintana, Keith Kuester, and Juan Rubio-Ramírez, "Fiscal volatility shocks and economic activity," American Economic Review, 2015, 105 (11), 3352-84.

Geweke, John and Gianni Amisano, "Hierarchical Markov normal mixture models with applications to financial asset returns," Journal of Applied Econometrics, 2011, 26 (1), 1-29.

Guerron-Quintana, Pablo A, "Common and idiosyncratic disturbances in developed small open economies," Journal of International Economics, 2013, 90 (1), 33-49.

Jacquier, Eric, Nicholas G Polson, and Peter E Rossi, "Bayesian analysis of stochastic volatility models," Journal of Business \& Economic Statistics, 2002, 20 (1), 69-87.

Jurado, Kyle, Sydney C Ludvigson, and Serena Ng, "Measuring uncertainty," American Economic Review, 2015, 105 (3), 1177-1216.

Justiniano, Alejandro and Bruce Preston, "Can structural small open-economy models account for the influence of foreign disturbances?," Journal of International Economics, 2010, 81 (1), 61-74.

Kim, Sangjoon, Neil Shephard, and Siddhartha Chib, "Stochastic volatility: likelihood inference and comparison with ARCH models," The review of economic studies, 1998, 65 (3), 361-393.

Koop, Gary, M Hashem Pesaran, and Simon M Potter, "Impulse response analysis in nonlinear multivariate models," Journal of econometrics, 1996, 74 (1), 119-147.

Koopman, Siem Jan and Eugenie Hol Uspensky, "The stochastic volatility in mean model: empirical evidence from international stock markets," Journal of applied Econometrics, 2002, 17 (6), 667-689.

McCausland, William J, "The HESSIAN method: Highly efficient simulation smoothing, in a nutshell," 
Journal of Econometrics, 2012, 168 (2), 189-206.

_ , Shirley Miller, and Denis Pelletier, "Simulation smoothing for state-space models: A computational efficiency analysis," Computational Statistics E Data Analysis, 2011, 55 (1), 199-212.

Mertens, Karel and Morten O Ravn, "A reconciliation of SVAR and narrative estimates of tax multipliers," Journal of Monetary Economics, 2014, 68, S1-S19.

Mumtaz, Haroon and Francesco Zanetti, "The impact of the volatility of monetary policy shocks," Journal of Money, Credit and Banking, 2013, 45 (4), 535-558.

_ and Konstantinos Theodoridis, "The international transmission of volatility shocks: An empirical analysis," Journal of the European Economic Association, 2015, 13 (3), 512-533.

_ and _ , "The changing transmission of uncertainty shocks in the US," Journal of Business ES Economic Statistics, 2017, pp. 1-14.

_ and _ , "Common and country specific economic uncertainty," Journal of International Economics, 2017, $105,205-216$.

Pitt, Michael K and Neil Shephard, "Filtering via simulation: Auxiliary particle filters," Journal of the American statistical association, 1999, 94 (446), 590-599.

Rue, Håvard, Sara Martino, and Nicolas Chopin, "Approximate Bayesian inference for latent Gaussian models by using integrated nested Laplace approximations," Journal of the royal statistical society: Series $b$ (statistical methodology), 2009, 71 (2), 319-392.

Schmitt-Grohé, Stephanie, "The international transmission of economic fluctuations:: Effects of US business cycles on the Canadian economy," Journal of international Economics, 1998, 44 (2), 257-287. 